\title{
Time evolution of gaps in stellar streams in axisymmetric Stäckel potentials
}

\author{
Helmer H. Koppelman ${ }^{1,2}$ and Amina Helmi ${ }^{1}$ \\ 1 Kapteyn Astronomical Institute, University of Groningen, Landleven 12, 9747 AD Groningen, The Netherlands \\ 2 School of Natural Sciences, Institute for Advanced Study, Princeton, NJ 08540, USA \\ e-mail: koppelman@ias.edu
}

\begin{abstract}
Context. When a subhalo interacts with a cold stellar stream it perturbs its otherwise nearly smooth distribution of stars, and this leads to the creation of a gap. The properties of such gaps depend on the parameters of the interaction. Their characterisation could thus lead to the determination of the mass spectrum of the perturbers and possibly reveal the existence of dark subhalos orbiting the Milky Way.

Aims. Our goal is to construct a fully analytical model of the formation and evolution of gaps embedded in streams orbiting in a realistic Milky Way potential.

Methods. To this end, we extend the model of Helmi \& Koppelman (2016) for spherical potentials, and predict the properties of gaps in streams evolving in axisymmetric Stäckel potentials. We make use of action-angles and their simple behaviour to calculate the divergence of initially nearby orbits slightly perturbed by the interaction with a subhalo.

Results. Our model, corroborated by N-body experiments, predicts that the size of a gap grows linearly with time. We obtain analytical expressions for the dependencies of the growth rate on the orbit of the stream, the properties of the subhalo (mass, scale-radius), and the geometry of the encounter (relative velocity, impact parameter). We find that the density at the centre of the gap decreases with time as a power-law in the same way as the density of a stream. This results in the density-contrast between a pristine and a perturbed stream on the same orbit asymptotically reaching a constant value dependent only on the encounter parameters.

Conclusions. We find that at a fixed age, smallish gaps are sensitive mostly to the mass of the subhalo, while gaps formed by subhalo flybys with a low relative velocity, or when the stream and subhalo move parallel, are degenerate to the encounter parameters.
\end{abstract}

Key words. Galaxy: halo - Galaxy: kinematics and dynamics - Galaxy: structure - dark matter

\section{Introduction}

The widely accepted $\Lambda \mathrm{CDM}$ model is very successful in reproducing the large-scale structure of the Universe (e.g. Davis et al. 1985), but it faces some key problems on small scales (e.g. Bullock \& Boylan-Kolchin 2017). For example, on the scales of individual galaxies, we observe much less substructure than what is predicted by dark matter only cosmological simulations (Klypin et al. 1999; Moore et al. 1999). Such simulations show that substructure exists down to very small scales and can be found at all radii, although preferentially in larger numbers in the outskirts of galaxies' halos (e.g. Diemand et al. 2008; Springel et al. 2008).

There exist several possibilities to solve this missing substructure conundrum. For example, adding baryonic physics to the simulations alleviates some of the problems, although mostly in the inner part of galaxies (e.g. D'Onghia et al.2010; Zhu et al. 2016; Sawala et al. 2017). Adjusting the properties of the dark matter particle (e.g. self-interacting dark matter, warm dark matter, or fuzzy dark matter) can help in suppressing the formation of the smallest substructures (e.g. Spergel \& Steinhardt 2000, Hu et al. 2000; Bode et al. 2001; Vogelsberger et al. 2016| Bozek et al. 2016; Hui et al. 2017). Another solution is to assume that the structures are present but in a dark form. Dark structures only reveal their presence through gravitational interaction, rendering them very difficult to detect. Results from gravitational lensing support the existence of dark structures at a level that is compat- ible with $\Lambda$ CDM (Dalal \& Kochanek 2002; Vegetti et al.|2010 2012, Ritondale et al. 2019: Hsueh et al. 2020).

Establishing whether there exists a population of subhalos with masses $<10^{8} \mathrm{M}_{\odot}$ in and around the Galaxy is therefore of the utmost importance as it can lead to a better understanding of the nature of the dark matter particle. Clearly, the discrepancy between the predicted and observed small-scale structure could be hinting at a fundamental problem with our current cosmological paradigm.

In this work, we will focus on a method to indirectly detect dark subhalos in our own Galaxy, through their possible interactions with cold stellar streams. Such streams are thin, almost one-dimensional elongated structures consisting of stars that originate from the tidal disruption of globular clusters or small dwarf galaxies. Because of their fragile nature, these streams are easily perturbed by gravitational interactions, making them promising probes of dark substructures (Ibata et al. 2002, Johnston et al. 2002). Occasional flyby's of dark subhalos can lead to the creation of a gap in an otherwise relatively smooth distribution of stars (Yoon et al. 2011; Carlberg 2013). Unfortunately, finding streams is challenging because of their low surface brightness, let alone finding gaps in streams. However, recent deep photometric surveys have identified a few dozen of narrow streams (e.g. Belokurov et al. 2006, Bernard et al.|2016, Shipp et al. 2018). The analysis of Gaia DR2 Gaia Collaboration et al. (2016, 2018) has also yielded another dozen streams (Malhan et al.|2018, Ibata et al. 2019). So far, only two of 
these streams have been claimed to contain gaps: GD-1 (Grillmair \& Dionatos 2006) and Palomar 5 (or Pal 5) (Odenkirchen et al. 2001), although several other streams show peculiarities (Bonaca et al. 2019a; Shipp et al. 2019; Li et al. 2020).

GD-1, is a promising stream to probe for gaps because of its length and coldness. It is known to contain several non-smooth features (Carlberg \& Grillmair 2013; , De Boer et al. 2018, PriceWhelan \& Bonaca 2018). The origin of these features, or gaps, is currently highly debated in the literature. For example, they could have been formed by an interaction with a massive (dark) object of $10^{6}-10^{8} \mathrm{M}_{\odot}$ that might have once been part of the Sagittarius system (Bonaca et al. 2019b, 2020, see also Banik et al. 2019). On the other hand, it has been argued that the presence and nature of a nearly periodic spatial distribution of gaps is an indication that these could be explained by internal dynamics without the need to recur to interactions with dark structures (Ibata et al.2020).

Pal 5's stream has been tentatively shown to host two gaps and several other features that would be consistent with being induced by subhalos in the range of $10^{6}-10^{8} \mathrm{M}_{\odot}$ (Erkal et al. 2017; Bovy et al. 2017). The inferred number of interactions appears to agree with the expected number predicted by CDM-only simulations (e.g. Sanderson et al. 2016). Unfortunately, Pal 5's stream is not ideally suited to look for gaps due to dark structures because of its proximity to the Galactic Centre. The high baryon density in this region can lead to the formation of irregularities in the stream's profile, for example, due to interactions with the bar (Pearson et al. 2017), globular clusters and with other baryonic structures (Banik \& Bovy 2018). Moreover, some of the gaps and features found in Pal 5's stream may be explained by survey incompleteness (Thomas et al.2016).

Since the expectation is that in the near future many gaps in many different streams will be detected, it is imperative to develop an in-depth understanding of the characteristics and evolution of these gaps. With such an understanding we may be able to link the population of gaps to an underlying population of dark substructures. For example, we need to establish the relation between subhalos and gap sizes, the growth rate of gaps and the dependence of their properties on the encounter parameters as well as on the characteristics of the host potential. Clearly, the ultimate goal would be to infer the properties of the perturbers from the analysis of the gaps observed.

Erkal \& Belokurov (2015a) developed a framework that predicts the evolution of gaps formed in streams that are orbiting on circular orbits. Using this model, Erkal \& Belokurov (2015b) showed how to infer the properties of a subhalo from the properties of a gap, down to a degeneracy in subhalo mass and relative velocity. A more recent model by Sanders et al. (2016) focuses on modelling gaps in angle-frequency space, allowing for eccentric orbits (see also Bovy et al. 2017). The authors validate several, but not all aspects of Erkal \& Belokurov (2015a), and argue for example that the velocity dispersions in the underlying stream affect the evolution of the gap, and thus should be taken into account. A caveat of all these models is that they are not fully analytical - and thus always rely on numerical exploration of the parameter space - or they are limited to circular orbits only. For this reason, we presented a fully analytical model for the evolution of gaps in streams (Helmi \& Koppelman 2016. HK16 hereafter) orbiting in spherical potentials.

In this work, we extend the HK16 model to streams orbiting in axisymmetric potentials. The model presented here not only predicts the behaviour of the size of the gap as a function of time but also its central density and their dependence on the characteristic parameters of the encounter. This paper is structured as follows. In Sec.2 we describe the model in detail and its predictions for the properties of gaps. In Sec. 3 we validate our model with N-body experiments. Subsequently, in Sec. 4 we analyse the dependencies of the gap's properties on the collision parameters and investigate possible degeneracies in the parameters. Finally, we present a discussion and conclusions in Sec. 5 .

\section{Methods}

The main reason to extend our HK16 model, which only works for spherical potentials, is that the Milky Way is more realistically described as an axisymmetric system. From a dynamical point of view, breaking the spherical symmetry will add a degree of freedom to the system.

The notation we use here is very similar to that employed in HK16. It builds on the action-angle stream description of Helmi \& White (1999. HW99 hereafter), see also Helmi \& Gomez (2007).

\subsection{Choice of the potential}

We are somewhat restricted in our choice for a potential for the Milky Way because our approach is based on the use of actionangle variables. These can only be calculated in potentials that are separable in the coordinates. For this reason, we will use Stäckel potentials, which are separable in ellipsoidal coordinates and are fully integrable (in fact, they are the only type of potentials with this property).

Because the (inner part of the) Milky Way is best described as an oblate system, we will use a set of prolate spheroidal coordinates $(\lambda, \phi, \nu)$ which we adopt from de Zeeuw (1985). The coordinate $\phi$ is the azimuthal angle and the other two coordinates, $\lambda$ and $v$, are the roots for $\tau$ in

$$
\frac{R^{2}}{\tau+\alpha}+\frac{z^{2}}{\tau+\gamma}=1
$$

where $R=x^{2}+y^{2}$, and $\alpha$ and $\gamma$ are constants related to the shape of the spheroid. The most general form of a Stäckel potential in these coordinates is

$\Phi(\lambda, v)=\frac{(v+\gamma) G(v)-(\lambda+\gamma) G(\lambda)}{\lambda-v}$,

where $G(\tau)$ determines the exact shape of the potential. For $G(\tau)$ we choose a two-component Kuzmin-Kutuzov potential, which takes the following form

$G(\tau)=\frac{G M_{h}}{\sqrt{\tau}+c_{h}}+\frac{G M_{d}}{\sqrt{\tau-q}+c_{d}}$,

where $q$ is a parameter set by the choice of the different axis ratios for the components taking into account the constraint that the sum remains a Stäckel potential: $\lambda_{h}-v_{h}=\lambda_{d}-v_{d}$, or $\lambda_{d}=$ $\lambda_{h}-q$ and $v_{d}=v_{h}-q$, where

$q=c_{h}^{2} \frac{\epsilon_{h}^{2}-\epsilon_{d}^{2}}{1-\epsilon_{d}^{2}}, \quad$ with $q \geq 0$.

Here the ratio of the semi-major $a$ and semi-minor $c$ axis $\epsilon^{2}=$ $\alpha / \gamma$ (i.e. the flattening of the system) is a free parameter for each component, where $\alpha=-a^{2}$ and $\gamma=-c^{2}$. Finally, we define the fraction of the mass of the disc with respect to the total mass as $k=M_{d} / M_{\mathrm{tot}}$, with $M_{\mathrm{tot}}=M_{d}+M_{h}$. We recommend the 


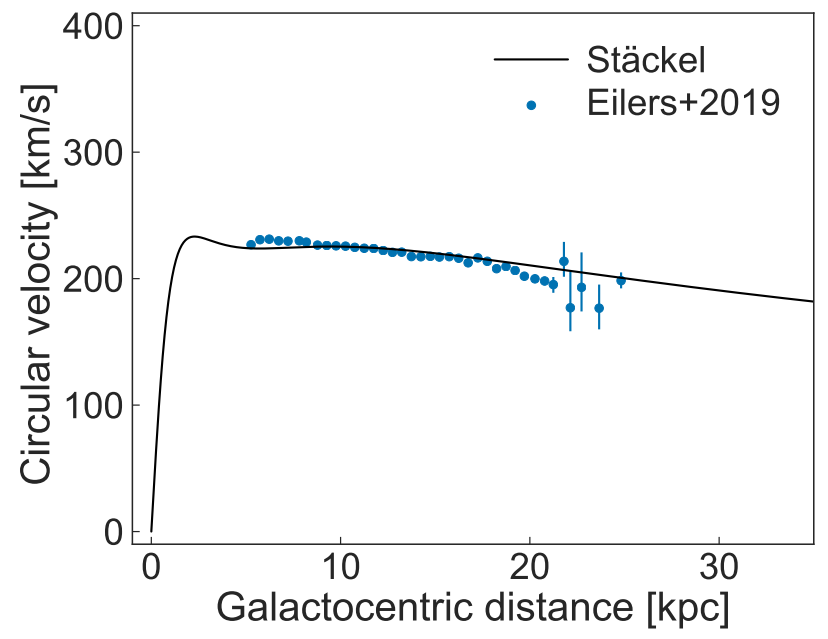

Fig. 1. Circular velocity in the plane of the disc of our Milky Way model. The Stäckel potential comprises a disc and a halo component and realistically describes the circular velocity of the Milky Way in the inner $\sim 20 \mathrm{kpc}$ as can be seen by comparing to the determinations by Eilers et al. (2019).

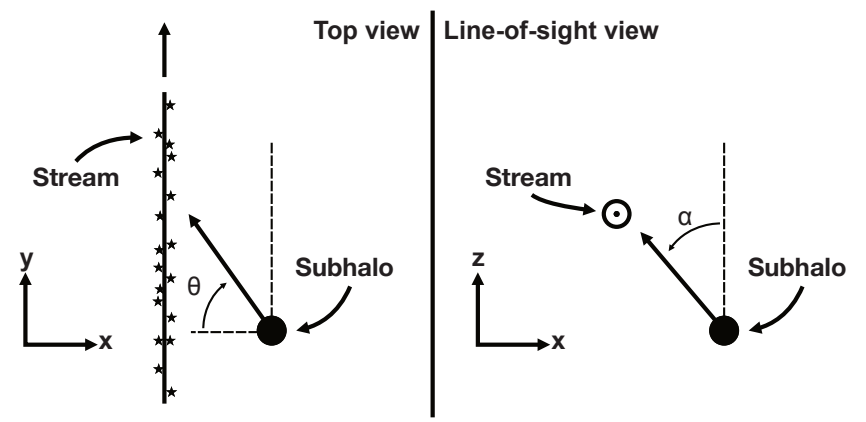

Fig. 2. Schematic overview of the stream-subhalo interaction. Left: view seen from the top. Right: view seen along the line of sight (stream moves into the paper).

interested reader to consult Dejonghe \& de Zeeuw (1988) for more details on axisymmetric Stäckel potentials.

The resulting potential is therefore described by five parameters, namely the total mass $M_{\text {tot }}$, the fraction of mass in the disc $k$, the scale length of the halo component $a_{h}$, and the flattening parameters of the halo $\epsilon_{h}$ and disc $\epsilon_{d}$. Here we set these parameter values to: $M_{\text {tot }}=4.0 \cdot 10^{11} \mathrm{M}_{\odot}, k=0.11, a_{h}=7.0 \mathrm{kpc}$, $\epsilon_{h}=1.02, \epsilon_{d}=75.0$ (which are based on Batsleer \& Dejonghe 1994: Famaey \& Dejonghe 2003, interested readers might want to consult also Reino et al. 2020, where two-component Stäckel potentials are fit to several streams around the Milky Way using Gaia DR2). The resulting potential matches reasonably well the circular velocity curve of the Milky Way, as can be seen from Fig. 1 (solid black line). This can be inferred by comparison to the recently estimated circular velocity curve from Eilers et al. (2019) (in blue).

\subsection{Impulse approximation}

Before diving into the model, we will first describe the impact that a subhalo has on a cold stream. The gravitational interaction of a subhalo is well described by the impulse approximation 11 (Yoon et al. 2011, Carlberg 2013). We de-

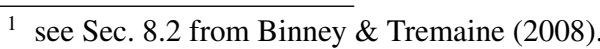

fine a reference system where the stream is aligned along the $y$-axis, and moves in the positive $y$-direction (similar to the system of Erkal \& Belokurov 2015a, c.f. their Fig. 2). In this co-moving frame the relative velocity vector of the subhalo is $\boldsymbol{w}=w(-\cos \theta \sin \alpha, \sin \theta, \cos \theta \cos \alpha)$, or $\boldsymbol{w}=$ $\left(-w_{\perp} \sin \alpha, w_{\|}, w_{\perp} \cos \alpha\right)$, where $w_{\perp}=w \cos \theta$ and $w_{\|}=w \sin \theta$. Figure 2 illustrates the geometry of the stream-subhalo encounter.

The change of velocity (i.e. the impulse) of a particle along the stream due to the encounter is

$\Delta v_{i}=\int_{-\infty}^{\infty} a_{i}\left(\boldsymbol{x}, \boldsymbol{w}, M, r_{s}\right) \mathrm{d} t$,

where $i=(x, y, z)$. The acceleration $a_{i}$ is a function of the relative velocity $\boldsymbol{w}$, the distance to the point of impact $y$, and of the subhalo mass $M$ and scale radius $r_{s}$. We model the subhalos as Plummer spheres but the expressions can be generalised for other profiles (Sanders et al. 2016). The change in velocities in all three coordinates at the time of the impulse according to Eq. (5) is

$$
\begin{aligned}
& \frac{\Delta v_{x}}{2 G M}=\frac{y w_{\perp} w_{\|} \sin \alpha}{w\left(r_{s}^{2} w^{2}+y^{2} w_{\perp}^{2}\right)}=\frac{y \cos \theta \sin \theta \sin \alpha}{w\left(r_{s}^{2}+y^{2} \cos ^{2} \theta\right)}, \\
& \frac{\Delta v_{y}}{2 G M}=-\frac{w_{\perp}^{2} y}{w\left(r_{s}^{2} w^{2}+y^{2} w_{\perp}^{2}\right)}=-\frac{y \cos ^{2} \theta}{w\left(r_{s}^{2}+y^{2} \cos ^{2} \theta\right)}, \\
& \frac{\Delta v_{z}}{2 G M}=-\frac{y w_{\perp} w_{\|} \cos \alpha}{w\left(r_{s}^{2} w^{2}+y^{2} w_{\perp}^{2}\right)}=-\frac{y \cos \theta \sin \theta \cos \alpha}{w\left(r_{s}^{2}+y^{2} \cos ^{2} \theta\right)} .
\end{aligned}
$$

The above expressions are valid for direct encounters, that is when the impact parameter $b=0$. Eqs. (1-3) in Erkal \& Belokurov (2015a) provide a more general form for the velocity changes which take into account the parameter $b$. This parameter enters into the equations above through $r_{s}^{2} \rightarrow r_{s}^{2}+b^{2}$.

We assume that the stream is linear over the scale where the impulse is significant. Moreover, the equations above assume that that the stream is a $1 \mathrm{D}$-structure. This approximation is sufficient when the width of the stream is smaller than the scale radius of the subhalo. However, the expressions can be generalised to the full 3D case, for which we find

$\Delta v_{i}(\boldsymbol{x})=-\frac{2 G M}{w} \frac{w^{2} x_{i}-w_{i}(\boldsymbol{x} \cdot \boldsymbol{w})}{\left(r_{s}^{2}+\boldsymbol{x} \cdot \boldsymbol{x}\right) w^{2}-(\boldsymbol{x} \cdot \boldsymbol{w})^{2}}$,

with $i=(x, y, z)$. To gain insight into the model we will use the equations of the 1D approximation in this section. However, when evaluating the model we will use the full 3D equations.

From Eq. (6) we can find the maximum kick in velocities $\Delta v_{i}^{\max }$ and at what distance $y_{\max }$ to the centre of impact it occurs,

$\Delta v_{i}^{\max }=-\frac{2 G M}{w} \frac{w^{2} x_{i}-w_{i} w_{y} y_{\max }}{\left(r_{s}^{2}+y_{\max }^{2}\right) w^{2}-\left(y_{\max } w_{y}\right)^{2}}$,

where $x_{i}=\left[0, y_{\max }, 0\right]$ and

$y_{\max }=\frac{w r_{s}}{\sqrt{w^{2}-w_{y}^{2}}}=\frac{r_{s}}{\cos \theta}$.

Typical profiles of $\Delta v_{y}(y)$ are shown and discussed in Sec. 3, see Fig. 11 . 


\subsection{Action-Angle variables}

This section aims to serve as a brief introduction to these variables, and it is by no means exhaustive or comprehensive. For more details on action-angle variables, the reader could consult Goldstein et al. (2002); Binney \& Tremaine (2008).

Orbits in smooth and simple potentials (e.g. spherical, axisymmetric, triaxial) have a number of integrals of motion: properties that do not change in time and serve to characterise them. For a spherical potential, the integrals of motion are the total energy (or the Hamiltonian) and the angular momentum vector. Orbits in axisymmetric systems (e.g. disc galaxies) typically have up to three integrals of motion: the total energy, the momentum in the azimuthal direction, and a non-classical integral which in most cases does not take an analytic form.

For separable potentials (e.g. the Stäckel potentials discussed in Sec. 2.1 there exist three isolating integrals $\mathbf{J}$, known as the actions. Each action is paired with a conjugate coordinate $\boldsymbol{\Theta}$, the angles. Together, these coordinates make up the action-angle variables $(\boldsymbol{\Theta}, \mathbf{J})$. The actions uniquely define the orbit, that is, a point in action-space corresponds to a complete orbit in phasespace. The conjugate angles define the phase, that is they specify where along the orbit a body is located at any given time.

To obtain the action-angle variables we make use of the Hamiltonian $H$, which being an integral of motion must depend on the actions (i.e. $H=H(\mathbf{J})$ ). The rate of change of the angles $\dot{\boldsymbol{\Theta}}=\partial H / \partial \mathbf{J}$ is known as the frequency $\mathbf{\Omega}(\mathbf{J})$. Therefore

$\boldsymbol{\Theta}(t)=\boldsymbol{\Theta}_{0}+\boldsymbol{\Omega} t$,

and hence the angles are linearly dependent on time. Finally, the actions $\mathbf{J}$ of a bound orbit in a separable potential are defined as

$\mathbf{J}=\frac{1}{2 \pi} \oint \mathbf{p} \cdot \mathrm{d} \mathbf{q}$

where $(\mathbf{q}, \mathbf{p})$ are any set of generalised phase-space coordinates and momenta.

\subsection{Size of the gap using an actions-angles framework}

The analytical framework of the method that we will use to describe the evolution of a gap in a stream with time, was first established by HW99. Originally, this framework was used to describe the divergence in the orbits of a distribution of nearby particles. It makes use of a linearised Taylor expansion around a central orbit. In our case, we will model the size of the gap as the spatial separation of two orbits: one on each side of the gap. These orbits are taken to be those of the particles that receive the largest impulse from the subhalo flyby. In practice, this is equivalent to modelling the (size of the) gap as twice the separation of the central orbit and one of the edges of the gap, as gaps are symmetric with respect to their centre.

\subsubsection{Generalities}

Let us consider a central orbit and some other orbit separated by $\Delta \mathbf{X}_{0}$ and $\Delta \mathbf{V}_{0}$, where the subscript is used to denote the time of the impact between the subhalo and the stream, $t=t_{0}$. To calculate the evolution of this separation vector we first transform it to action-angle variables

$\left[\begin{array}{c}\Delta \boldsymbol{\Theta}_{0} \\ \Delta \mathbf{J}_{0}\end{array}\right]=\mathcal{M}_{0}\left[\begin{array}{l}\Delta \mathbf{X}_{0} \\ \Delta \mathbf{V}_{0}\end{array}\right]$ where $\mathcal{M}_{0}$ is a matrix calculated at $t=t_{0}$ that locally transforms from Cartesian coordinates to action-angles. In practice, the transformation is a product of matrices

$\mathcal{M}_{0}=\mathcal{M}_{0}^{\mathrm{AA} \leftarrow \mathrm{st}} \mathcal{M}_{0}^{\mathrm{st} \leftarrow \mathrm{cyl}} \mathcal{M}_{0}^{\mathrm{cyl} \leftarrow \mathrm{xyz}}$,

where $\mathcal{M}_{0}^{\beta \leftarrow \alpha}$ transforms the set of coordinates $\alpha$ to the set $\beta$, xyz indicating Cartesian coordinates, cyl cylindrical coordinates, st spheroidal coordinates used for the Stäckel potential, and $A A$ action-angle variables.

Next, the separation vector in action-angles can be evolved in time by expanding linearly Eq. (9) and making use of the matrix $\Omega^{\prime}$

$\Omega^{\prime}=\left[\begin{array}{cc}I_{3} & \partial \mathbf{\Omega} / \partial \mathbf{J} t \\ 0 & I_{3}\end{array}\right]$,

At any point in time, the separation in action-angle coordinates can be transformed back to Cartesian coordinates locally, and therefore

$\left[\begin{array}{l}\Delta \boldsymbol{X}_{t} \\ \Delta \boldsymbol{V}_{t}\end{array}\right]=\mathcal{M}_{t}^{-1} \Omega^{\prime} \mathcal{M}_{0}\left[\begin{array}{l}\Delta \mathbf{X}_{0} \\ \Delta \mathbf{V}_{0}\end{array}\right]$,

where $\mathcal{M}_{t}^{-1}$ is the (local) transformation back to Cartesian coordinates at time $t$ and at the location of the central orbit of the gap.

Finally, the size of the gap can be taken as twice the separation calculated in Eq. (14). The initial separation of the two orbits describing the gap can be obtained assuming Eq. 8a and Eq. (8b). Since the two orbits are typically separated a few kpc initially, we need to add the velocity gradient of the orbit to the separation in velocities, so $\Delta V_{0}=\Delta v^{\max }+\delta v_{\text {orbit }}$. We note that this is an $a d$ hoc fix to the non-local nature (finite extent) of the stream. It takes into account that the velocity of the stream particles changes as a function of location.

\subsubsection{Long-term behaviour}

The growth rate of the size of the gap can be derived from Eq. (14) in a similar fashion as shown in HK16. In the limit where $t \gg t_{0}$ (or better $t / t_{\text {orb }} \gg 1$ ), this equation simplifies to

$\left[\begin{array}{l}\Delta \boldsymbol{X}_{t} \\ \Delta \boldsymbol{V}_{t}\end{array}\right] \sim t\left[\begin{array}{l}\mathcal{M}_{t, 1}^{-1} \partial \mathbf{\Omega} / \partial \mathbf{J} \Delta \mathbf{J}_{0} \\ \mathcal{M}_{t, 3}^{-1} \partial \mathbf{\Omega} / \partial \mathbf{J} \Delta \mathbf{J}_{0}\end{array}\right]$

where $\mathcal{M}_{t, 1}^{-1}$ is the upper left submatrix of $\mathcal{M}_{t}^{-1}$, and $\mathcal{M}_{t, 3}^{-1}$ is the bottom left submatrix. The spatial separation of the two orbits is equal to the length of vector $\Delta \boldsymbol{X}_{t}$

$\left|\Delta \boldsymbol{X}_{t}\right|=\sqrt{\Delta \boldsymbol{X}_{t}^{\dagger} \Delta \boldsymbol{X}_{t}} \sim t \sqrt{\Delta \mathbf{J}_{0}^{\dagger} f_{x, \boldsymbol{\Omega}} \Delta \mathbf{J}_{0}}$.

In this equation $f_{\boldsymbol{x}, \boldsymbol{\Omega}}=\partial \mathbf{\Omega} / \partial \mathbf{J} \mathcal{M}_{t, 1}^{-1^{\dagger}} \mathcal{M}_{t, 1}^{-1} \partial \mathbf{\Omega} / \partial \mathbf{J}$. Similarly, we can calculate the velocity difference of the two orbits $\Delta \boldsymbol{V}$

$\left|\Delta \boldsymbol{V}_{t}\right|=\sqrt{\Delta \boldsymbol{V}_{t}^{\dagger} \Delta \boldsymbol{V}_{t}} \sim t \sqrt{\Delta \mathbf{J}_{0}^{\dagger} f_{\boldsymbol{v}, \mathbf{\Omega}} \Delta \mathbf{J}_{0}}$,

where $f_{\boldsymbol{v}, \mathbf{\Omega}}=\partial \mathbf{\Omega} / \partial \mathbf{J} \mathcal{M}_{t, 3}^{-1^{\dagger}} \mathcal{M}_{t, 3}^{-1} \partial \mathbf{\Omega} / \partial \mathbf{J}$.

We note that the terms $f_{\boldsymbol{x}, \boldsymbol{\Omega}}$ and $f_{\boldsymbol{v}, \boldsymbol{\Omega}}$ are dependent on the orbit of the gap and its location, but they do not depend on the impact parameters. Both $\left|\Delta \boldsymbol{X}_{t}\right|$ and $\left|\Delta \boldsymbol{V}_{t}\right|$ are linearly dependent on time $t$, similar to gaps orbiting in spherical potentials. Interestingly, the ratio of the two separations is constant with time which potentially can be used to infer the properties of the gap at any time (as we will demonstrate also in Sec. 4). 


\subsection{Density of stream gaps}

We now build further on the framework developed by HW99 and focus on modelling the evolution of the density of the gap. The impulse imparted on the stream by the subhalo increases the local velocity dispersion of the stars in the gap. This causes it to grow faster and thus appear as under dense region in comparison to the neighbouring parts of the stream. If we know the central orbit of the gap and the initial phase-space distribution around it, we can calculate the evolution of the density in the gap.

\subsubsection{Generalities}

We will describe this initial phase-space distribution as a multivariate Gaussian distribution, in other words

$f(\boldsymbol{x}, \boldsymbol{v})=f_{0} \exp \left(-\frac{1}{2} \Delta_{\varpi, 0}^{\dagger} \sigma_{\varpi, 0} \Delta_{\varpi, 0}\right)$,

where $f_{0}$ is the phase-space density at $t=t_{0}, \Delta_{\varpi, 0}$ is a separation vector: $\Delta_{\varpi, 0}=\xi_{i}-\xi_{c, i}$ and where $\xi_{i}=\left[x, y, z, v_{x}, v_{y}, v_{z}\right]$ and $\xi_{c, i}$ is the central point of the distribution (which we will take to be the location where the subhalo impacts the stream, or in the terminology previously used, the central orbit) at $t=t_{0}$. The matrix $\sigma_{\varpi, 0}$ is the inverse of the covariance matrix of the phasespace coordinates.

To compute the initial dispersion matrix of the gap, we start from the original, unperturbed distribution and add the impulse in the velocities according to Eq. (7). That is, we transform $\sigma_{\varpi, 0}^{\text {stream }}+$ impulse $\rightarrow \sigma_{\varpi, 0}^{\text {gap }}$. Below we show how to calculate the new covariance matrix in the regime where the stream is approximated by a 1D-structure, but in Appendix A we provide the full 3D expressions.

The most general form of the initial unperturbed covariance matrix $\Sigma_{\varpi, 0}$ is

$\Sigma_{\varpi, 0}=\sigma_{\varpi, 0}^{-1}=\left(\begin{array}{ccc}\sigma_{x}^{2} & C(x, y) & \cdots \\ C(y, x) & \sigma_{y}^{2} & \cdots \\ \vdots & \vdots & \ddots\end{array}\right)$

where $C(x, y)$ is the covariance of $x$ and $y$ and $\sigma_{x}$ is the standard deviation of $x$. The covariance matrix can be represented with $3 \times 3$ block matrices

$\Sigma_{\varpi, 0}=\left(\begin{array}{ll}\mathbb{C}_{x, x} & \mathbb{C}_{x, v} \\ \mathbb{C}_{v, x} & \mathbb{C}_{v, v}\end{array}\right), \quad$ where $\quad \mathbb{C}_{x, v}=\mathbb{C}_{v, x}^{\dagger}$

We now proceed to compute the perturbed covariance matrix by computing the changes of each individual element due to the encounter with the subhalo. The impulse only affects the velocities. Therefore, the position block matrix $\left(\mathbb{C}_{x, x}\right)$ does not change during the encounter. The first element with a velocity term is in the $\mathbb{C}_{\boldsymbol{x}, \boldsymbol{v}}$ block matrix

$C\left(v_{x}, x\right)=\frac{1}{n} \sum_{i=1}^{n}\left(v_{x_{i}}-\mu_{v_{x}}\right)\left(x_{i}-\mu_{x}\right)$,

where $n$ is the total number of particles, $\mu_{v_{x}}$ and $\mu_{x}$ are the mean $v_{x}$ and $x$ of the distribution in the region around the gap. After applying the impulse, the new covariance element becomes

$C\left(v_{x}+\Delta v_{x}, x\right)=\frac{1}{n} \sum_{i=1}^{n}\left(v_{x_{i}}+\Delta v_{x_{i}}-\mu_{v_{x}}-\Delta \mu_{v_{x}}\right)\left(x_{i}-\mu_{x}\right)$, where $\Delta v_{x_{i}}$ is the velocity change of particle $i$, and $\Delta \mu_{v_{x}}$ is the shift of the mean velocity of all particles. Since the kicks are symmetric around the central point, the mean shift of velocities is zero $\Delta \mu_{v_{x}}=0$, and we can rewrite the covariance term as

$C\left(v_{x}+\Delta v_{x}, x\right)=C\left(v_{x}, x\right)+\frac{1}{n} \sum_{i=1}^{n} \Delta v_{x_{i}}\left(x_{i}-\mu_{x}\right)$.

Considering that the covariance matrix describes the central density (i.e. positions close to the centre) we can express the kick $\Delta v_{x}$ as a function that is only linearly dependent on $y$, since the quadratic term in the denominator of Eq. 6a is negligible (i.e. $r_{s}^{2} w^{2}>y^{2} w_{\perp}^{2}$ ). Moreover, since the velocity kicks are calculated in a frame where there is symmetry with respect to $y$ (i.e. $\mu_{y}=0$ ) we can rewrite the last term in the equation above as

$\frac{1}{n} \sum_{i=1}^{n} \Delta v_{x_{i}}\left(x_{i}-\mu_{x}\right)=2 G M \frac{w_{\perp} w_{\|} \sin \alpha}{r_{s}^{2} w^{3}} \frac{1}{n} \sum_{i=1}^{n}\left(y_{i}-\mu_{y}\right)\left(x_{i}-\mu_{x}\right)$,

which is equal to

$C\left(v_{x}+\Delta v_{x}, x\right)=C\left(v_{x}, x\right)+2 G M \frac{w_{\perp} w_{\|} \sin \alpha}{r_{s}^{2} w^{3}} C(y, x)$.

The new covariance term $C\left(v_{x}+\Delta v_{x}, x\right)$ can be expressed as the old covariance term plus a new term that depends on the impact parameters. The procedure shown above can be extended to all covariance terms of the form $C\left(\alpha, v_{\beta}+\Delta v_{\beta}\right)$ and $C\left(v_{\beta}+\Delta v_{\beta}, \alpha\right)$, where $(\alpha, \beta)=(x, y, z)$.

Using similar arguments, it is easy to show that covariance terms in the velocity submatrix $\left(\mathbb{C}_{v, v}\right)$ take the following general form

$$
\begin{aligned}
C\left(v_{\alpha}+\Delta v_{\alpha}, v_{\beta}+\Delta v_{\beta}\right)= & C\left(v_{\alpha}, v_{\beta}\right)+C\left(\Delta v_{\alpha}, v_{\beta}\right) \\
& +C\left(v_{\alpha}, \Delta v_{\beta}\right)+C\left(\Delta v_{\alpha}, \Delta v_{\beta}\right) .
\end{aligned}
$$

For example, for $\alpha=x$ and $\beta=y$, and following similar procedures as above

$$
\begin{aligned}
& C\left(\Delta v_{x}, v_{y}\right)=2 G M \frac{w_{\perp} w_{\|} \sin \alpha}{r_{s}^{2} w^{3}} C\left(y, v_{y}\right), \\
& C\left(v_{x}, \Delta v_{y}\right)=-2 G M \frac{w_{\perp}^{2}}{r_{s}^{2} w^{3}} C\left(v_{x}, y\right), \\
& C\left(\Delta v_{x}, \Delta v_{y}\right)=-\left(2 \frac{G M}{r_{s}^{2} w^{3}}\right)^{2} w_{\perp}^{3} w_{\|} \sin \alpha C(y, y) .
\end{aligned}
$$

We now have full expressions for the matrix $\sigma_{\varpi, 0}$ in Eq. (18) representing the phase-space configuration around the gap at the time of the encounter $t=t_{0}$. By transforming $\sigma_{\varpi, 0}$ to actionangle coordinates as $\sigma_{\omega, 0}=\mathcal{M}_{0}^{-1 \dagger} \sigma_{\varpi_{0}} \mathcal{M}_{0}^{-1}$, where $\mathcal{M}$ is the transformation matrix defined in Eqs. (11) and (12), we can calculate the evolution in time of the covariance matrix in phasespace using Eq. (13). This allows us to describe the local density of the portion of the stream around the location of the impact by the subhalo (i.e. of the gap) as

$\rho\left(\boldsymbol{x}_{c}, t\right)=\int f\left(\boldsymbol{x}_{c}, \boldsymbol{v}, t\right) \mathrm{d}^{3} \boldsymbol{v}$,

where $\rho\left(\boldsymbol{x}_{c}, t\right)$ is the density of orbits in a location around the central orbit. In the principal axes, where the velocity covariance matrix is diagonal, this density takes a simple form

$\rho\left(\boldsymbol{x}_{c}, t\right)=\rho_{0} / \sqrt{\operatorname{det}\left|\sigma_{v}\right|} \propto \rho_{0} \sigma_{v_{1}}(t) \sigma_{v_{2}}(t) \sigma_{v_{3}}(t)$,

where $\rho_{0}$ is the central density at $t=t_{0}$ and $\sigma_{v_{1}}, \sigma_{v_{2}}, \sigma_{v_{3}}$ are the velocity dispersions along the three principal axes. 

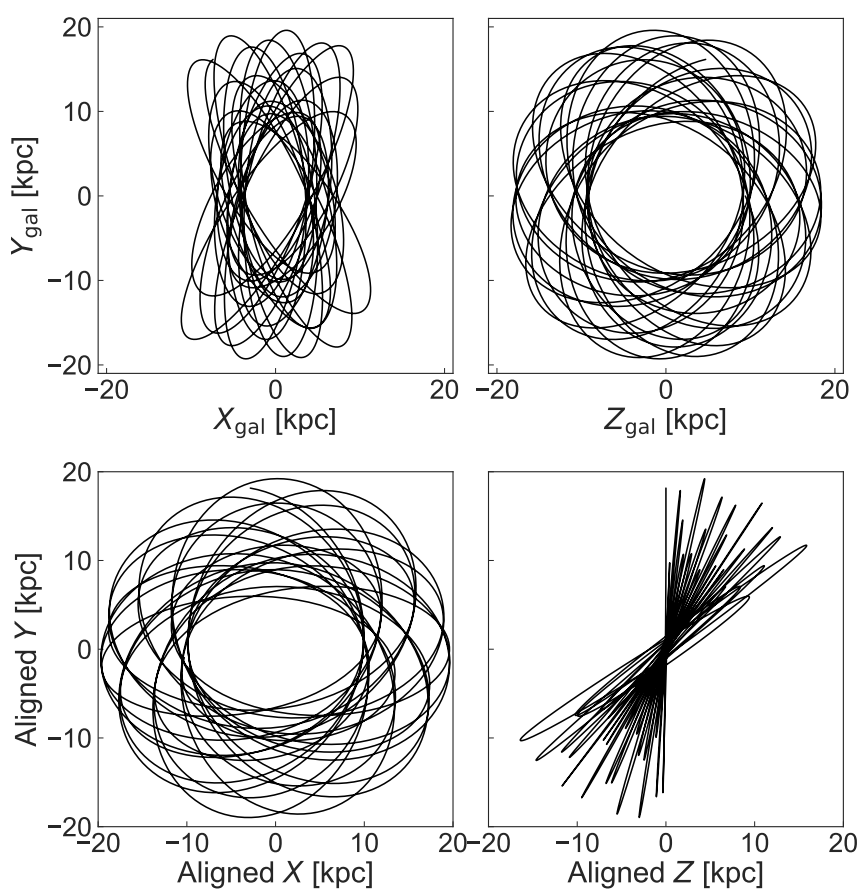

Fig. 3. The orbit of the stream shown in galactocentric Cartesian coordinates, evolved for $10 \mathrm{Gyr}$ forward in time in the Stäckel axisymmetric Milky Way-like potential. Top: orbit in coordinates aligned with the host galaxy (e.g. plane of the disc is $z=0$ ). Bottom: coordinates aligned with the initial angular momentum vector of the orbit. The right bottom panel highlights the precession of the orbital plane, which is indicative of the non-spherical nature of the potential considered.

\subsubsection{Long-term behaviour of the density}

Using the above formalism, it is possible to show that the density of a stream (and thus also that of a gap) decreases as a power law of time which depends on the number of degrees of freedom of the orbit of the stream (Vogelsberger et al. 2008)

$\rho \propto t^{-n}$, with $\mathrm{n}=$ d.o.f.

Ultimately these degrees of freedom are determined by the number of independent frequencies, and this number generally is dependent on the functional form of the potential. For axisymmetric galaxies the number of d.o.f. is 3 for most (non-resonant) orbits. On the other hand, for example, circular orbits only have one degree of freedom, implying that the density decreases much slower (i.e. $1 / t)$.

HW99 derived a general expression for the central density at late times for streams (and gaps) in a general Stäckel potential (see their Appendix C) and found

$\rho\left(\mathbf{x}_{c}, t\right)=\frac{\rho_{0} f_{\text {orb }}}{\sqrt{\operatorname{det}\left|\sigma_{\Theta_{0}}\right|}} t^{-3}$,

where $\rho_{0}$ is the initial density of the distribution, $f_{\text {orb }}$ is a constant determined by the central orbit, and $\sigma_{\Theta_{0}}$ is the angle submatrix at $t=t_{0}$. This implies that the ratio of the density of a perturbed to unperturbed stream is a constant

$\delta \rho_{\text {str }}^{\text {gap }}=\sqrt{\frac{\operatorname{det}\left|\sigma_{\Theta_{0}}\right|_{\text {str }}}{\operatorname{det}\left|\sigma_{\Theta_{0}}\right|_{\text {gap }}}}$

as all other variables are independent on the impact parameters. We will refer later in this work to this ratio of densities as the density contrast.
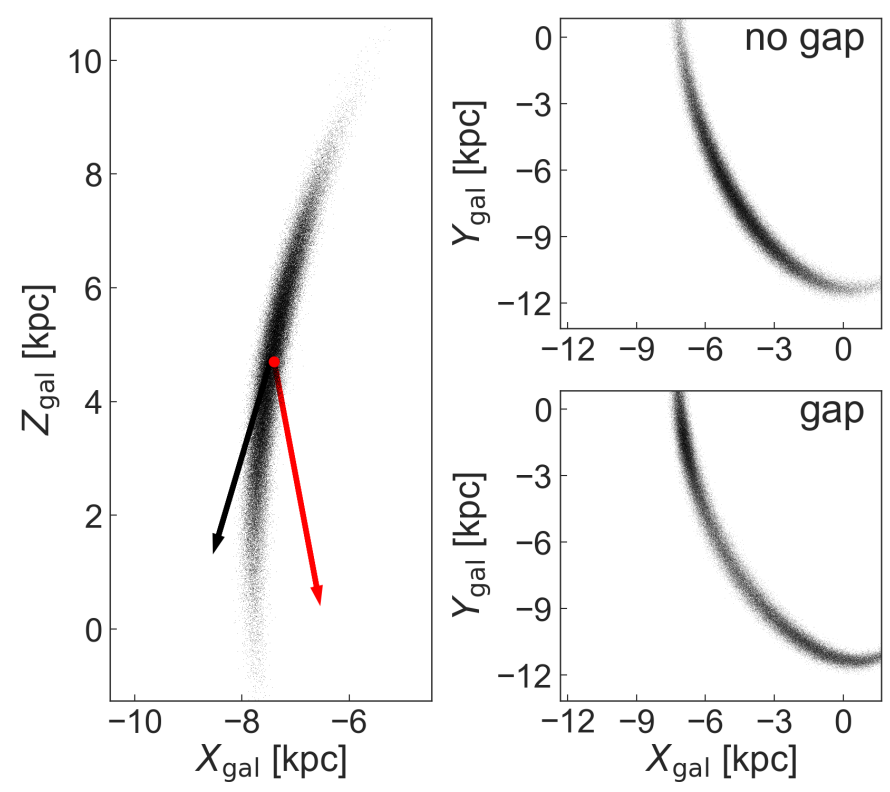

Fig. 4. Left: Snapshot of a stream at the time of interaction with a subhalo. The stream is plotted with black dots and the centre of mass of the subhalo is shown as a red solid circle. The velocity vector of the stream is marked with a black arrow and that of the subhalo with a red arrow. Right: Both panels show the stream after 2 Gyr of evolution, in isolation in the top panel, and after the encounter with the subhalo in the bottom panel.

Table 1. The masses and scale radii of the subhalos. The subhalos are modelled as rigid Plummer spheres.

\begin{tabular}{lccc}
\hline \hline & subhalo 1 & subhalo 2 & subhalo 3 \\
\hline $\mathrm{M}\left[\mathrm{M}_{\odot}\right]$ & $10^{6}$ & $10^{7}$ & $10^{8}$ \\
$\mathrm{r}_{s}[\mathrm{kpc}]$ & 0.35 & 0.59 & 1.35 \\
\hline
\end{tabular}

\subsection{Setting up the stream-subhalo encounter}

To verify our model predictions, we perform N-body simulations of the encounter of a subhalo with a stream orbiting in the Milky Way potential described in Sec. 2.1. To this end, we use a modified version of GADGET-2 (Springel 2005), where we model the host as the rigid potential, and the subhalo as a rigid Plummer sphere which is centred on a particle with a negligible mass that is put on a trajectory in the host potential.

The progenitor of the stream is modelled with $10^{6}$ test particles ${ }^{2}$ following a Gaussian distribution in $6 \mathrm{D}$ phase space, with $\sigma_{x}=0.2 \mathrm{kpc}$ and $\sigma_{v}=0.5 \mathrm{~km} / \mathrm{s}$. These very low dispersions are chosen such that the stream has a high density even a few Gyr after forming. In comparison, globular clusters orbiting the Milky Way typically have a $\sigma_{v}$ of a few km/s (e.g. Harris 1996. 2010 edition).

The progenitor is put on an elongated orbit with maximum distance from the centre $r_{\max } \approx 20 \mathrm{kpc}$ and minimum distance $r_{\min } \approx 10 \mathrm{kpc}$, reaching $r_{z}= \pm 20 \mathrm{kpc}$ above the plane of the disc, as shown in Fig. 3. After the progenitor of the stream is evolved for $1 \mathrm{Gyr}$ in the host potential, a subhalo is inserted on a trajectory that directly crosses the stream. We remove the subhalo after the collision to isolate a single interaction, and when its gravitational effect is sufficiently small that it no longer affects the stream.

2 Because we use test particles, it is not strictly necessary to model their evolution using an N-body code such as Gadget. 


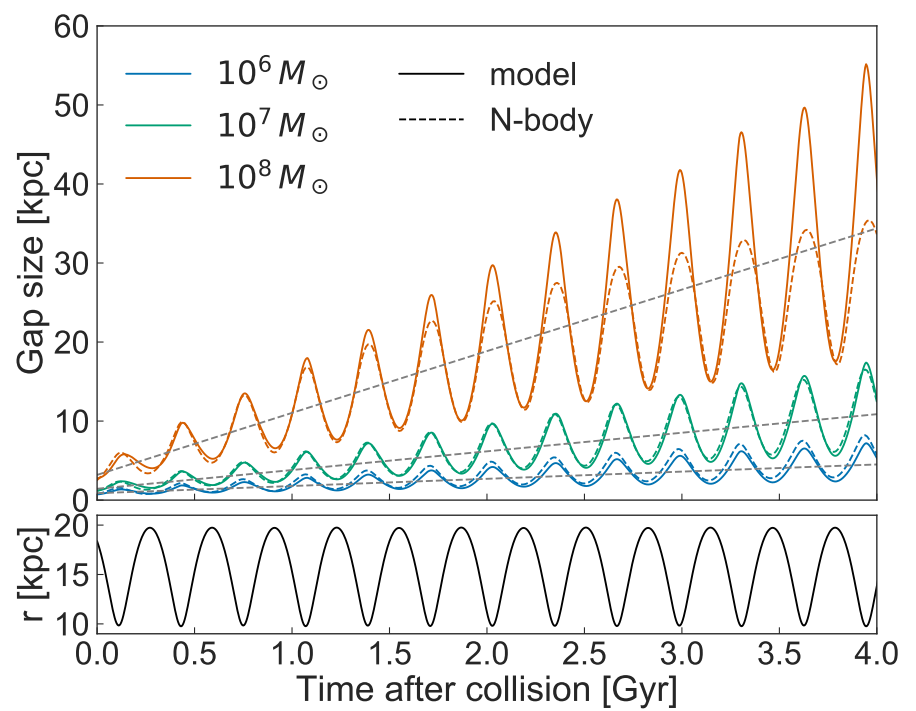

Fig. 5. Top panel: Gaps growing in a stream orbiting an axisymmetric Stäckel Milky Way-like potential. The lines with different colours show the size of the gaps induced by subhalos of different sizes. The solid curves correspond to our analytic model, while the dashed curves to gaps measured in the $\mathrm{N}$-body experiments. The straight grey dashed lines illustrate the linear growth rate of the gaps. Bottom panel: Distance of the central orbit to the centre of the host potential.
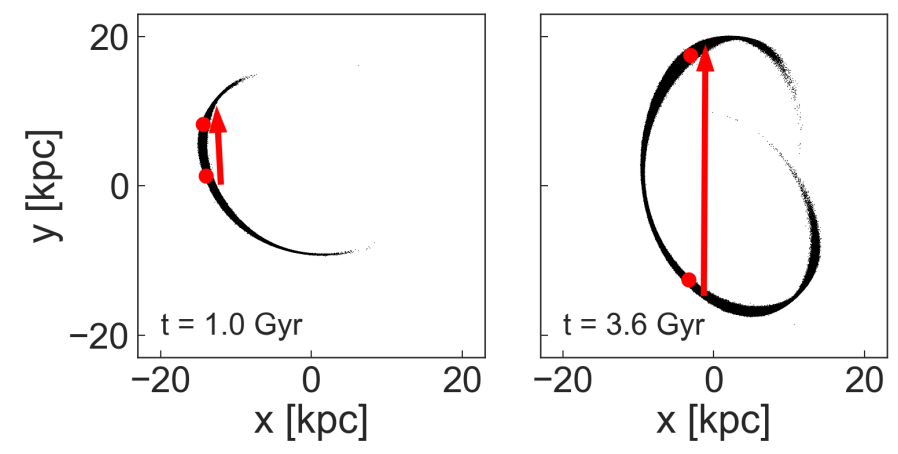

Fig. 6. Left: A stream 1 Gyr after an interaction with a subhalo. The two red dots indicate the orbits that are being used to measure the size of the gap, the red arrow shows the distance on a straight line between the two dots. Right: The same stream, 3.6 Gyr after the encounter. At this point in time the two orbits (red dots) are close to their maximum separation.

Fig. 4 shows an example of a stream-subhalo interaction. The left panel shows both the stream and a subhalo at the time of the collision. The right panels show a stream with and without an encounter, 2 Gyr after the interaction with the subhalo. The perturbed stream clearly shows a gap of several kpc in size at the centre of the panel.

\section{Results}

We compare the predictions of the model presented in Sec. 2.4 and Sec. 2.5 with the gaps produced in the N-body experiments. We will first investigate gaps produced by subhalos of varying mass and size for a fixed encounter configuration (i.e. the same velocity and impact angle). Next, we focus on the effects of a varying configuration while keeping the subhalo properties fixed.

\subsection{Size evolution}

Figure 5 shows the evolution of gaps caused by interactions sharing the same configuration, but with different subhalo masses (see Table 1 for their properties). The model (solid lines) reproduces very well the size of the gap as measured in the N-body simulation (coloured dashed lines). The latter is measured as the average separation of two groups of 50 particles on each side of the gap. These 50 particles are identified as those that experience the largest velocity change at the time of the impact. We use 50 particles to lower the effects of discreteness of the N-body simulation, but there is only very little difference when using the single particle with the maximum velocity change on each side of the gap. The bottom panel of Figure 5 shows the total distance of the gap to the centre of the host galaxy and gives an indication of its orbit. The frequency of $r(t)$ and the oscillations in the gap size are in antiphase. This is naturally expected since the gap will be stretched at pericentre and be smallest at apocentre.

Although Fig. 5] shows that the model reproduces the gap size in the N-body experiment very well, there appears to be an upper limit to its measured size. The largest difference is apparent for the encounter with the most massive subhalo at late times. This limit occurs because the size of the gap becomes comparable to the typical scale of the orbit and hence our method of measuring the size of the gap fails to work. The typical scale of the specific orbit that is used here is $\lesssim 40 \mathrm{kpc}$, see Fig. 6. This value can also be determined analytically using the inverse of Eq. (11), and considering that the two orbits on each side of the gap are at a maximum separation at $\Delta \Theta=\pi$. The maximum distance between two particles on the same orbit but apart by $180^{\circ}$ in the angles, at any location in the orbit, is $\sim 35 \mathrm{kpc}$. This value agrees very well with the ceiling reached by the red, dashed line measured from the N-body experiment in Fig. 5 .

The size of the gap in this regime is pushing the limits of our analytical model. The transformation from action-angles to Cartesian coordinates (i.e. Eq. 14p is only valid 'locally' near the central orbit, and therefore the approximation breaks down for such large gaps. Although it should be possible to extend the formalism to include such cases, this is not really necessary as there are no known streams with gaps of this size - nor is it likely to observe one such gap in the (near) future.

\subsection{Evolution of the density}

Now we compare the density as predicted by our model with the density measured in N-body experiments. For the latter we count the number of particles in a small volume in 6D space with $r<0.1 \mathrm{kpc}$ and $v<7.5 \mathrm{~km} / \mathrm{s}$. This velocity limit does not remove any particles from the stream at $t_{0}$ when the impact occurs, but it removes particles that may have drifted away (i.e. have a different orbital phase) at later times. The volume is centred on the central orbit, which is determined in a simulation of a stream with the same set-up, but without a subhalo interaction.

Figure 7 compares the predicted (solid lines) and measured density from the N-body simulation (dashed lines) for a stream with and without a gap. For the latter, we have simulated the exact same stream with and without an encounter with a subhalo. Although the peaks and troughs of the stream and the gap are always larger in our model, the figure shows that the model provides an excellent description of the N-body experiments. The small differences can be attributed to a resolution effect: in the $\mathrm{N}$-body experiments we measure the density in a finite volume, whereas the model computes a density at a single location in space. If the density were measured in a smaller volume in our 


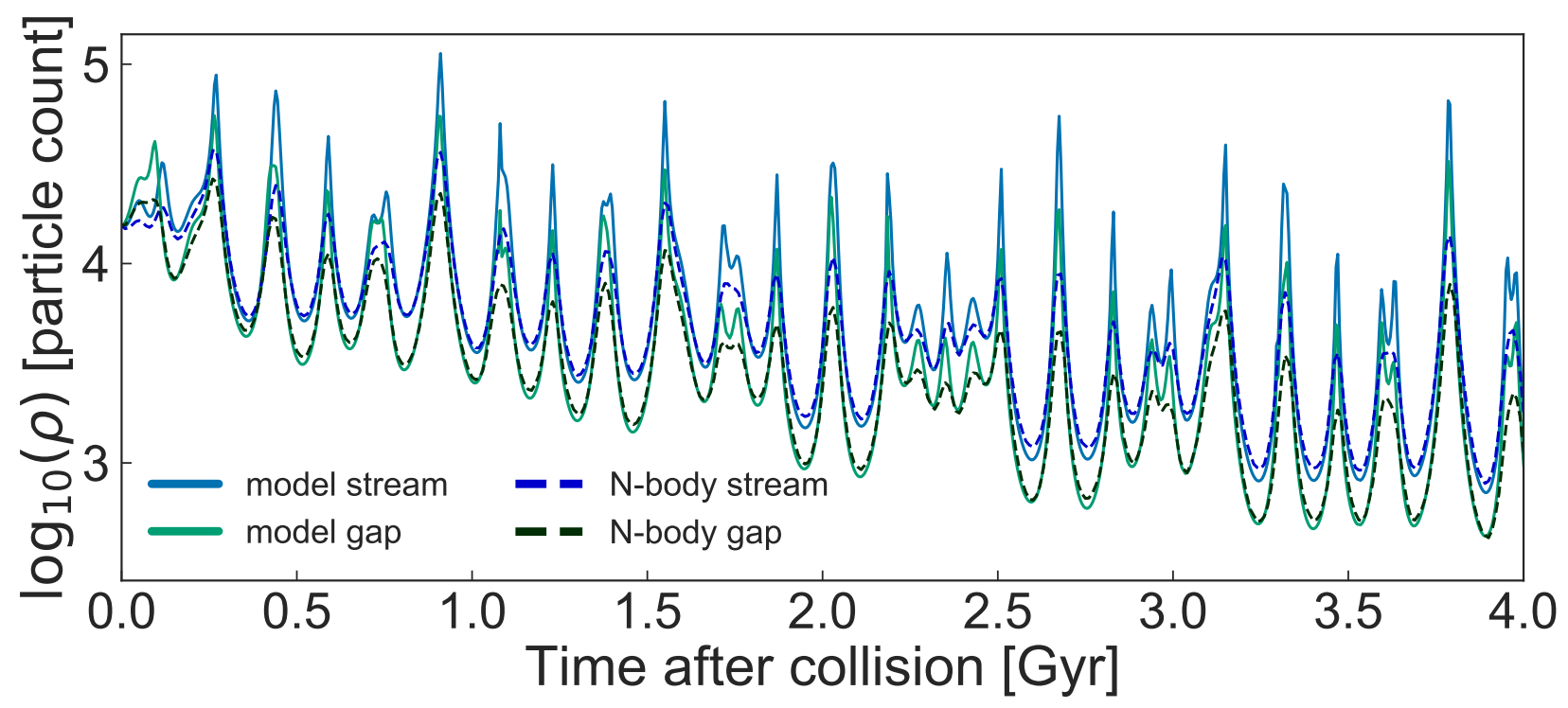

Fig. 7. Density evolution of an unperturbed stream (in blue) and of a gap in a stream following the same orbit (in green). The dashed curves are the densities obtained from the corresponding N-body experiments. The agreement between the solid and dashed curves is excellent. The subhalo that is used to create the gap in the stream has a mass of $10^{7} \mathrm{M}_{\odot}$.

experiments the peaks would be sharper. However, the number of particles would drastically decrease and drop to less than a handful in less than $4 \mathrm{Gyr}$ of evolution.

\subsubsection{Varying subhalo masses}

Next, we explore the evolution of the density contrast (i.e. the ratio of the density around the gap to that of the unperturbed stream) in Fig. 8. The figure shows the same experiments as those plotted in Fig. 5, with the density contrast of the most massive subhalo shown in a separate panel. For the most massive halo, we have modified slightly our set-up, instead of starting from the same initial conditions as the other experiments using the orbit shown in Fig. 3, we have used the location of the gap to determine its orbit. We used this as the central orbit both in our analytical model and for the N-body experiment representing the unperturbed stream. The reason for this is that when the subhalo and the stream interact, the stream receives an impulse that displaces it slightly from its original orbit. The effect is negligible for subhalos of $M \lesssim 10^{7} M_{\odot}$, and is small but apparent for more massive objects, particularly after $\sim 3-4 \mathrm{Gyr}$ of evolution. This new set-up is actually more realistic since when attempting to model an observed stream or gap, its actual measured position and velocity in a suitable gravitational potential would be integrated (as it is not possible to have a priori access to the original initial conditions of the orbit of the stream, before it received the impact).

In Fig. 8, we show with solid lines the predicted density contrast from our model, and with dashed lines those measured in the N-body experiments. The Poisson errors on the ratio of the densities as measured in the $\mathrm{N}$-body experiments are marked with shaded areas. In general, the amplitude of the density contrast is well reproduced by the model, with the difference in the amplitude of the narrow peaks at early times explained by the same resolution effects as described in Sec. 3.2.
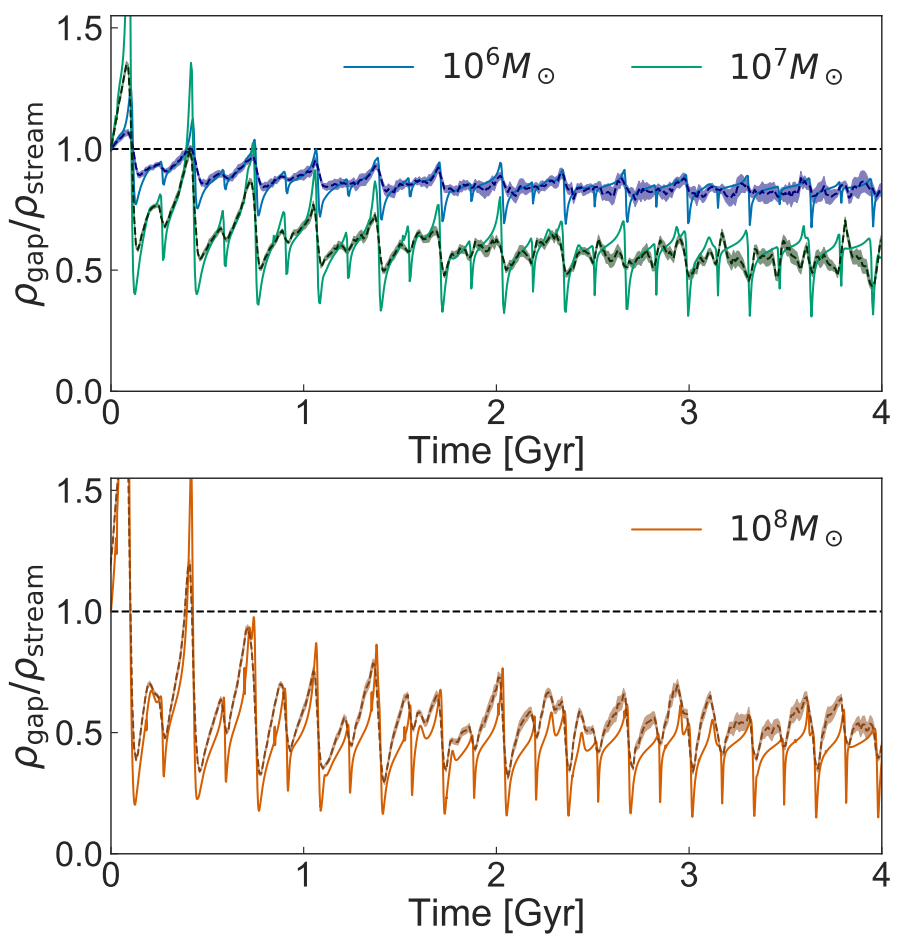

Fig. 8. Density contrast measured for a stream experiencing an encounter with subhalos of different mass and scale radius, see Table 1 . The relative velocity of the impact is the same for all three interactions. The coloured solid lines show the density contrast according to our model, and the dashed lines are measured in the N-body experiments. The shaded areas indicate the Poisson error in the observed density. The subhalo of $10^{8} \mathrm{M}_{\odot}$ is shown in a different panel because of its slightly different set-up.

\subsubsection{Variation of encounter configuration}

Finally, we check how our model performs for different configurations of the stream-subhalo encounter, keeping the subhalo at a fixed mass of $10^{7} \mathrm{M}_{\odot}$. We compare three different configurations which correspond to rotations of the same velocity vector, 


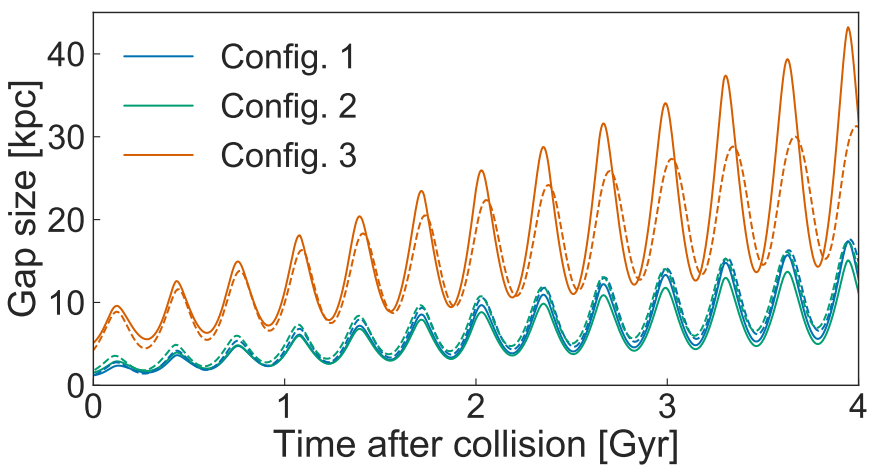

Fig. 9. Similar to Fig. 5 but for three different configurations of the stream-subhalo interactions. The gaps of all configurations are created by the same subhalo of size $10^{7} \mathrm{M}_{\odot}$, but their relative velocity $w$ and impact angles $\theta$ and $\alpha$ are different.

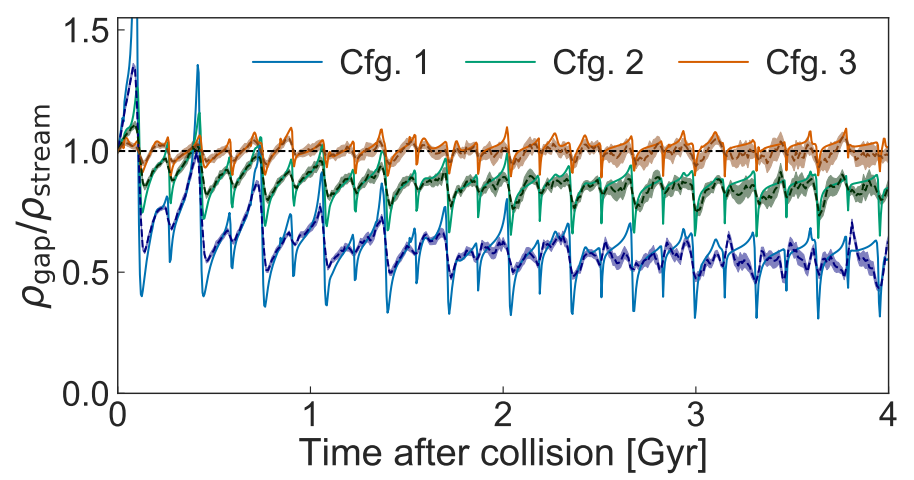

Fig. 10. Similar to Fig. 8 but for three different stream-subhalo interactions with a subhalo of fixed mass, and whose size evolution is described in Fig. 9

as listed in Table 2, with Configuration 1 being that used in the previous section. We note that the velocity vector is rotated in the rest frame, not in the co-moving frame of the stream. The resulting configurations thus have different velocity amplitudes in the co-moving frame.

Figures 9 and 10 show the time evolution of the size and density contrast. The layout of the figures is the same as in the previous section. Again, the model (solid lines) predicts the behaviour of the gaps as measured in the $\mathrm{N}$-body experiments (dashed lines) extremely well.

Interestingly, Configuration 2 with a subhalo of $10^{7} \mathrm{M}_{\odot}$ gives rise to a density contrast of similar amplitude as the collision with the subhalo of $10^{6} \mathrm{M}_{\odot}$ (see Fig. 8) on Configuration 1, both producing a gap with a density contrast of $\sim 0.9$. However, if we examine the size of the gaps we notice that the gap caused by the $10^{6} \mathrm{M}_{\odot}$ subhalo (blue curve Fig. 5) is smaller than that for the $10^{7} \mathrm{M}_{\odot}$. This implies that by measuring both size and density, one may be sensitive to different parameters characterising the encounter, as we shall discuss in more detail in Sec. 4.

Figure 9 shows that the gaps resulting from the encounter in Configurations 1 and 2 have initially approximately the same size. Interestingly the gap resulting in Configuration 3 is initially the largest and also remains the largest throughout its evolution in time (although the size is somewhat poorly modelled because of the change in velocities for this particular configuration is very shallow, see Fig. 11. which gives rise to some complications in identifying the correct particles to trace in the N-body).

On the other hand, Fig. 10 shows that the evolution of the density for Configuration 3 is very similar to that of an unper-

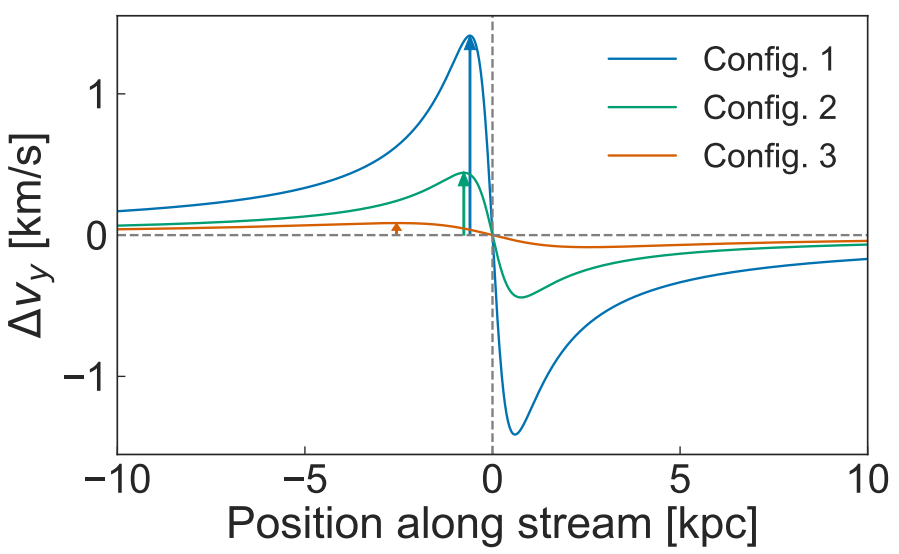

Fig. 11. Profile of the change in velocity along the stream $v_{y}$, for the three different configurations described in the two previous figures. In Configuration 1 the stream experiences the largest kick on the smallest scale. On the other hand, in Configuration 3 the kick is much smaller but on a much larger scale. The arrows indicate the location of the maximum $\Delta v_{y}$.

Table 2. Parameters of the different configurations, see Sec.2.2 for their definition.

\begin{tabular}{cccc}
\hline \hline & Config. 1 & Config. 2 & Config. 3 \\
\hline$\theta$ [deg.] & -10.5 & -34.1 & 97.6 \\
$\alpha$ [deg.] & 39.4 & 9.9 & 245.2 \\
$w$ [km/s] & 76.7 & 33.7 & 378.3 \\
\hline
\end{tabular}

turbed stream. To understand this consider the velocity kicks $\Delta v_{y}$ and their profiles as shown in Fig. 11. Since the initial size of the gap is derived from the location of the maximum velocity change, the gap produced in Configuration 3 initially is much larger than the other two. Comparing Fig. 10] and Fig. [11] we see that the steepest density contrast is associated with the largest change in velocity, exactly like one would expect. These results imply that the size of the gap is strongly correlated with the distance between the maximum velocity change, whereas the density contrast is more correlated with the amplitude of the change (see also the expressions in the next section).

\section{Exploration of the gap observables: dependencies and degeneracies}

Now that we have validated our analytic model, we will use it to explore how the size and density of a gap depend on the collision parameters using Eqs. (16) and (33). We will consider hypothetical gaps formed in the stream presented in Sec. 2.6 and analysed in Sec. 3. To this end, we vary the characteristic parameters of the collision with a subhalo, namely $w, \theta$, and $M$, while keeping the angle $\alpha$ fixed at some arbitrary value $\alpha=163^{\circ}$. We consider $w$ in the range $[0,800] \mathrm{km} / \mathrm{s}$ and $\theta$ in the range $\left[-90^{\circ}, 90^{\circ}\right]$. Instead of varying separately $M$ and $r_{s}$, we use a relation for $V_{\max } \propto r_{\max }$ for subhalos found in the 'Aquarius' simulations by Springel et al. (2008), see Appendix B for details.

Figure 12 shows the gap's properties, namely size and density contrast, as a function of these characteristic parameters. Each panel shows the dependence of these two observable quantities with one of the three parameters: $M, w$, or $\sin \theta$. At the same time, we vary discretely a second parameter, which gives rise to the different curves in each subpanel, but keep fixed the third parameter. For example, in the leftmost panels we show the variation of gap size (top) and density contrast (bottom) 2.5 Gyr 

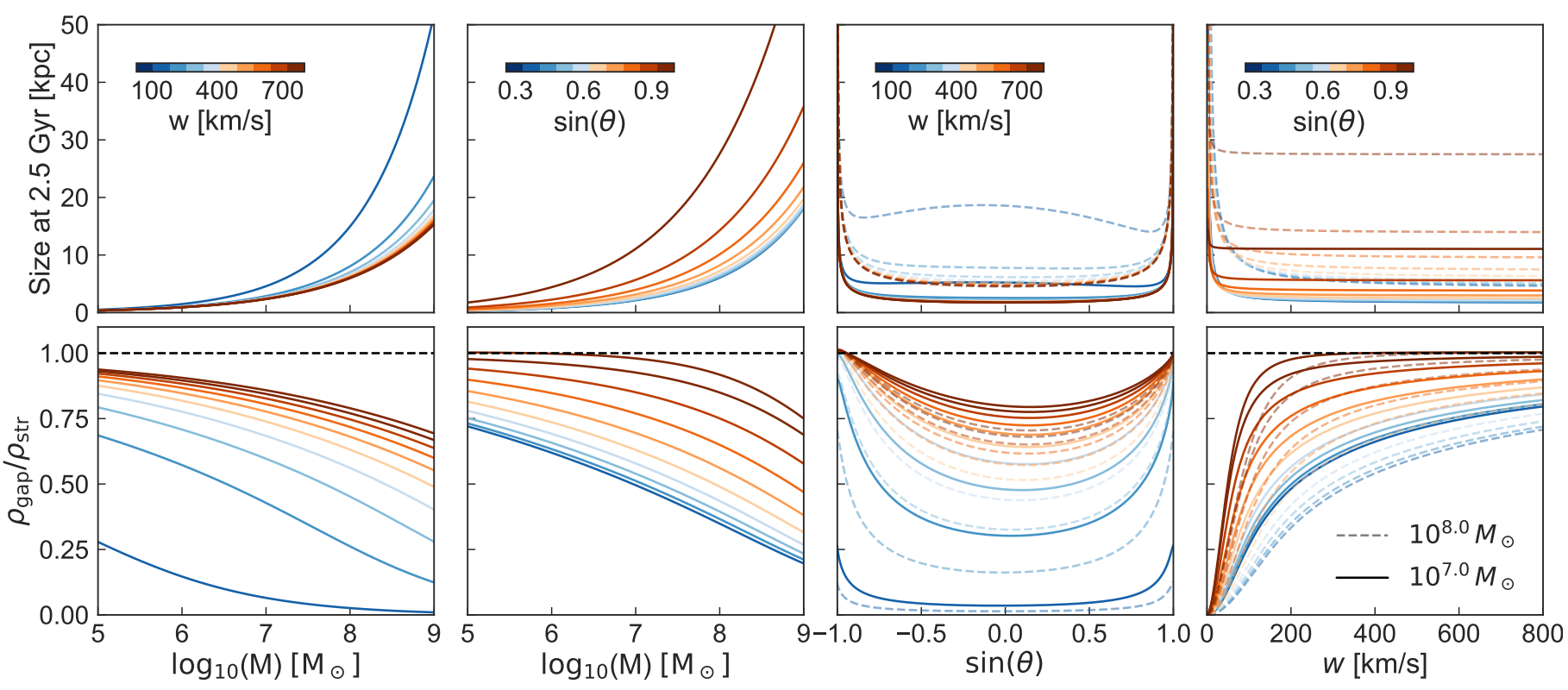

Fig. 12. Top row: size of the gap after $2.5 \mathrm{Gyr}$ of evolution, as a function of the impact parameters (subhalo mass, angle $\theta$ and amplitude of the relative velocity $w$ ). Bottom row: density contrast as a function of the same parameters. The two panels on the left show the discrete variation of the curves - varying the velocity amplitude $w$ or collision angle $\theta$. The two panels on the right show two sets of lines, solid for a subhalo of mass $10^{7} \mathrm{M}_{\odot}$ and dashed for $10^{8} \mathrm{M}_{\odot}$.

after impact as a function of mass of the subhalo $M$, for different values of $w$ as indicated by the colour bar, and for $\theta=\pi / 4$.

Since the size of a gap varies depending on its orbital phase, we have checked that the dependencies shown in Figure 12 are robust. We have found them to be identical, except for an overall scaling of the amplitude that depends on the phase. Since it will be possible to establish the phase of a gap observationally (after assuming a suitable Galactic potential and integrating the orbit of the stream in which it is embedded), this implies that the trends observed here can be used to infer several of the characteristic properties of the encounter. The density contrast, meanwhile, does not vary along the orbit (because the density variations along the orbit for the gap are identical to those for the unperturbed stream). However, in the bottom panels of Figure 12. we have taken the late times limit of the density contrast given by Eq. 34.

We have shown in Eq. $8 \mathrm{~b}$ that the size of the gap at any point in time strongly depends on its initial magnitude (i.e. $\propto$ $r_{s} / \cos \theta$ ) implying a dependence on the subhalo's mass through the $r_{s}$ parameter (with $r_{s} \propto M^{2 / 5}$ ), as shown in Appendix $\mathrm{B}$. This simple relation explains the curves in the top panels of Fig. 12 well, which show that the gap size depends strongly on the mass of the subhalo (two leftmost panels), with relatively little dependence on $w$ and $\sin \theta$, except for extreme values of these parameters (two rightmost panels). For example, when the subhalo moves along the stream (i.e. when $\cos \theta \rightarrow 0$ ) the size of the gap is clearly not well defined. In this case the impulse approximation breaks down as the subhalo and stream interact for a long time, and, perhaps more importantly, the interaction affects a large part of the stream. Also for very low values of $w$, the impulse approximation is no longer valid. Low relative velocities and extreme alignment must be rare because they only happen when the stream and subhalo move at a similar velocity and in the same direction. In summary, the top panels of Figure 12 suggest that given a gap size, it is possible to infer with some con- fidence the mass of the subhalo that perturbed it for most values of $w$ and $\theta$.

With knowledge of the mass, the density contrast could be used to infer some plausible encounter geometries. To understand the factors driving the density contrast, we use Eq. (34), which depends on the ratio of $\operatorname{det}\left|\sigma_{\Theta_{0}}\right|$ for the stream and the gap. Although general analytic expressions can be obtained, these are somewhat cumbersome. Under the assumption that the velocity kick is small (compared to the characteristic orbital velocity of the subhalo), and that the covariance matrix of the stream at the time of the encounter is diagonal in Cartesian coordinates, we find (see Appendix C for full details)

$\delta \rho_{\text {str }}^{\text {gap }} \propto 1-\frac{G M}{r_{s}^{2} w} f\left(\theta, \alpha, \mathbb{C}_{\boldsymbol{x}, \boldsymbol{x}_{0}}^{\mathrm{str}}, \mathbb{C}_{\boldsymbol{v}, \boldsymbol{v}_{0}}^{\mathrm{str}}, \boldsymbol{x}_{0}, \boldsymbol{v}_{0}\right)$,

where $f\left(\theta, \alpha, \mathbb{C}_{\boldsymbol{x}, \boldsymbol{x}_{0}}^{\mathrm{str}}, \mathbb{C}_{\boldsymbol{v}, \boldsymbol{v}_{0}}^{\mathrm{str}}, \boldsymbol{x}_{0}, \boldsymbol{v}_{0}\right)$ is a function that depends on the angles characterising the encounter, the location of the encounter $\left(\boldsymbol{x}_{0}, \boldsymbol{v}_{0}\right)$ and the configuration and velocity covariance matrices of the unperturbed stream at the time of the impact (see also Eq. C.16). This relation implies that the density contrast becomes shallower with increasing $w$, as can indeed be seen in Fig. 12. On the other hand, more massive subhalos create gaps with lower densities. Because of the different dependence of the gap size (top panel) and of the density contrast with the characteristic parameters of the encounter, $(M, w, \sin \theta)$, this means that it is possible to break some of the degeneracies present using these two observable quantities, provided the time since the collision could be established (which is necessary for making use of the constraints provided by gap size).

Another time-invariant combination of observables is plotted in Fig. 13. This figure shows the spatial size of the gap relative to the separation in velocity space, normalised by its initial value at $t_{0}$. Note that although the ratio of $\Delta X$ and $\Delta V$ does vary with the orbital phase of the gap/stream, this phase can be established through orbit integrations as discussed earlier. The lines shown in Fig. 13 (measured at $2.5 \mathrm{Gyr}$ ) are for a gap that is near its 

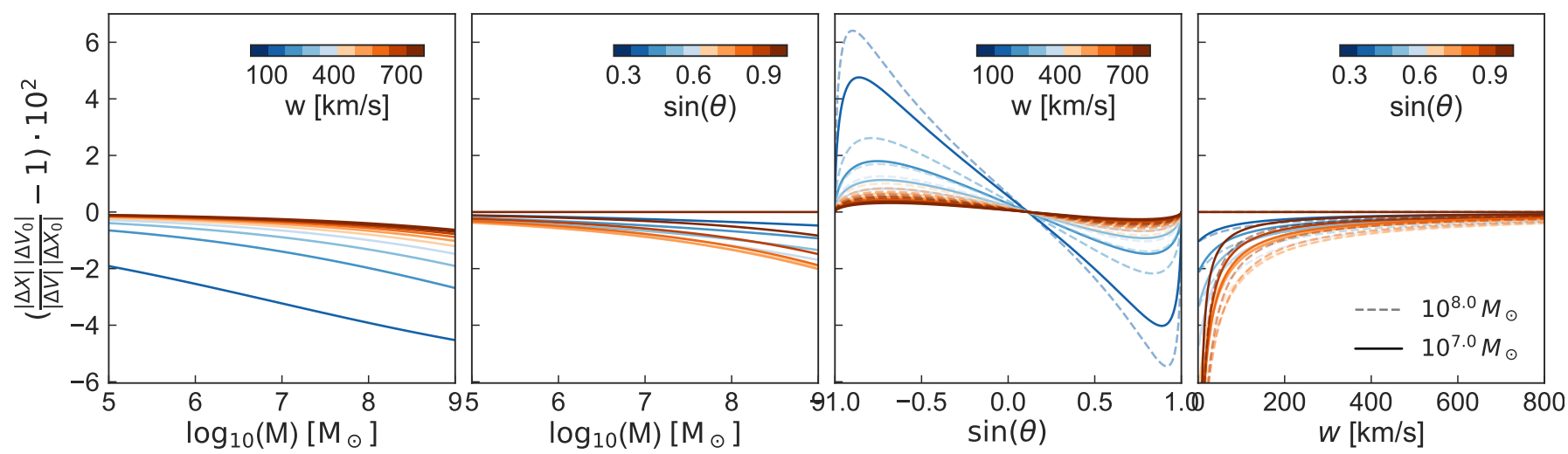

Fig. 13. Sensitivity of the (time-independent) ratio of the size of the gap to the separation in velocity to the same parameters discussed in Fig. 12

apocentre. Evaluating the ratios $\Delta X / \Delta V$ near the pericentre results in a similar figure, but where the $y$-axis is mirrored with respect to the line $y=0$.

There are clear similarities between this ratio and the behaviour of the density contrast shown in the bottom row of Fig. 12, except for the third panel, which reveals a sensitivity for low $w$ velocity on the angle of the encounter $\theta$. Overall, this ratio will therefore be a good discriminator for low $w$ of $\sin \theta$.

In summary (smallish) gaps $\lesssim 25 \mathrm{kpc}$ are mostly dependent on the mass of the subhalo, while large gaps can either be due to a specific configuration (low relative velocity or angle of the encounter) or due to a large subhalo. Assuming the average encounter has a relative speed of $w>200 \mathrm{~km} / \mathrm{s}$ it appears that per orbit/stream we can break the degeneracy of the interaction parameters using also the density contrast. We have checked that these conclusions (and dependencies) are robust and independent of the orbital characteristics of the stream (e.g. different inclinations with respect to the Galactic plane), but they are only strictly valid if the age of the gap can be well constrained.

\section{Discussion}

Action-angle variables have been previously used to describe streams and their gaps (e.g. Helmi et al. 1999; Helmi \& Gomez 2007; Bovy 2014; Sanders et al. 2016; Helmi \& Koppelman 2016; Bovy et al. 2017). There is a trade-off to be made when using these variables: one may either make use of a numerical approach and obtain a (local) approximation for a generic potential (Binney 2012, Sanders \& Binney 2015), or use a fully analytic approach and be restricted in the choice of the potential. In this work, we take the latter approach such that we can express the properties of the gaps in physical space directly as a function of the encounter parameters.

In contrast to the work of Erkal \& Belokurov (2015a), who argue that gaps grow at late times as $\sqrt{t}$ (for circular orbits), we find that both in our numerical experiments as in the analytic model, gaps grow linearly with time independently of the type of orbit or shape of the gravitational potential. We have thus extended the findings of HK16 who considered a spherical potential, and confirm also the results of Sanders et al. (2016).

Sanders et al. (2016) have found that the density contrast of a gap approaches a constant value at late times. Our fully analytical model allows us to verify their conclusion and we are also able to show why this happens and what the constant value depends upon (e.g. Eqs. 34 and 35). Sanders et al. (2016) also find that gaps grow differently in the leading and trailing arm.
Judging from the expressions derived in this work, there may be two reasons for the different growth rate: $i$ ) a difference in the local (velocity) dispersions of the particles in either the leading or trailing arm, as was already noted by Sanders et al. (2016); $i$ i) the orbits of the leading and trailing stream have slightly different characteristic parameters (they are slightly offset in energy), and these affect the growth rate of gaps as well as the decline in their density.

Erkal \& Belokurov (2015b) argue that there exists a degeneracy in the gap parameters with mass and velocity. The reported degeneracy of $(M, \mathbf{w}) \rightarrow(\lambda M, \lambda \mathbf{w})$ only exists if the scale radius $r_{s}$ of the subhalo is kept fixed, but its mass is not. For example, the size of a gap depends on $r_{s}$, while the density contrast depends on $M / r_{s}^{2}$. Since a non-linear relation between $r_{s}$ and $M$ is known to exist for subhalos in cosmological simulations ( $r_{s} \sim M^{2 / 5}$, Neto et al. 2007; Springel et al. 2008), we must conclude that the above degeneracy does not exist.

The model presented in this work successfully describes gaps in streams in axisymmetric potentials. However, it builds on several key assumptions, namely:

- The time of the collision/age of the gap. Although this information is in principle encoded in the size of the gap, we have generally explored its properties at a fixed time. Keeping it open will add one more parameter to optimise. A rough estimate of the formation time could be obtained by integrating the two sides of the gap backwards in time and to see when they meet. Recall on the other hand, that if we may assume that the encounter happened sufficiently long ago, the density contrast is time- independent, and some of the encounter parameters can be constrained.

- The potential of the host galaxy. Changes in the host potential will change the central orbit of the gap and thus the size and density evolution. However, we note that the explicit time dependence of both the size and density of the gap will not change with (small) variations in the potential.

- Knowledge of the pristine stream conditions, before the interaction. In principle from observing the full stream morphology and knowing the age of the gap, it should be possible to derive the full $6 \mathrm{D}$ properties of the stream at the time of the collision.

- The properties of the subhalo, here assumed to be welldescribed by a Plummer sphere. This choice was made because of its simple mathematical expression, for which there is an analytic solution to the integral of the impulse approximation used to compute the velocity kicks. However, it is 
possible to compute this integral numerically for other profiles (e.g. Sanders et al. 2016).

We have shown here that some of the previously reported degeneracies in the space of parameters describing the encounter can be broken by making plausible assumptions on the streamsubhalo configuration. A natural next step would be to consider probability distributions for the encounter parameters, much like, for example Erkal et al. (2016). Our model can then be used to quickly explore the parameter space as it can constrain the most likely encounter parameters given observation of a gap.

\section{Conclusions}

We have successfully extended the model of the evolution of gaps in spherical potentials, presented in HK16, to describe gaps in streams orbiting in axisymmetric Stäckel potentials. The model accurately predicts the evolution of both the size of the gap and its central density. The model is unique in that it is fully analytic, meaning that we can directly relate the stream-subhalo interaction parameters to the properties of the resulting gaps. In doing so, it provides some interesting insights into the evolution of gaps in streams.

We find that the sizes of the gaps in axisymmetric potentials grow linearly in time - and this dependence is independent of the shape of the Galactic potential. On the other hand, the density declines in time as $t^{-n}$ where $n$ denotes the number of independent frequencies characterising its orbit. The growth of the size and density of a gap depend on the subhalo properties (mass and scale radius), the properties of the stream at the time of the impact (velocity and positional differences of the particles), and on the central orbit of the gap.

We have shown that the size of the gap is correlated with the portion of the stream most affected by the subhalo flyby (the value $y_{\max }$ in the impulse approximation). The density contrast of the gap, on the other hand, is more correlated with the amplitude of the interaction $\left(\Delta v^{\max }\right)$. These different correlations are in the end, what drives the ability to break the degeneracy of the encounter parameters. For example, for a given gap age, small gaps $(<25 \mathrm{kpc})$ are very dependent on the size of the subhalo, while a large gap can be caused by a large subhalo, or by an alignment of the orbit of the stream and subhalo. These results are encouraging and appear to be useful to constrain the properties of a population of dark subhalos if present in the halo of the Milky Way.

Acknowledgements. We gratefully acknowledge financial support from a VICI grant and a Spinoza Prize from the Netherlands Organisation for Scientific Research (NWO) and HHK is grateful for the support from the Martin A. and Helen Chooljian Membership at the Institute for Advanced Study. We thank Tim de Zeeuw for his comments on an early version of this manuscript and Hans Buist for modifications made to the code used here. For the analysis, the following software packages have been used: vaex (Breddels \& Veljanoski 2018), numpy (Van Der Walt et al. 2011), matplotlib (Hunter 2007), jupyter notebooks (Kluyver et al.2016), f2py (Peterson 2009), and pyGadgetReader (Thompson 2014).

\section{References}

Banik, N. \& Bovy, J. 2018, MNRAS, 484, 2009

Banik, N., Bovy, J., Bertone, G., Erkal, D., \& de Boer, T. J. L. 2019, arXiv, 1911.02662

Batsleer, P. \& Dejonghe, H. 1994, A\&A, 287, 43

Belokurov, V., Zucker, D. B., Evans, N. W., et al. 2006, ApJ, 642, L137

Bernard, E. J., Ferguson, A. M. N., Schlafly, E. F., et al. 2016, MNRAS, 463, 1759
Binney, J. 2012, MNRAS, 426, 1324

Binney, J. \& Tremaine, S. 2008, Galactic dynamics (Princeton University Press), 885

Bode, P., Ostriker, J. P., \& Turok, N. 2001, ApJ, 556, 93

Bonaca, A., Conroy, C., Hogg, D. W., et al. 2020, ApJ, 892, L37

Bonaca, A., Conroy, C., Price-Whelan, A. M., \& Hogg, D. W. 2019a, ApJ, 881, L37

Bonaca, A., Hogg, D. W., Price-Whelan, A. M., \& Conroy, C. 2019b, ApJ, 880, 38

Bovy, J. 2014, ApJ, 795

Bovy, J., Erkal, D., \& Sanders, J. L. 2017, MNRAS, 466, 628

Bozek, B., Boylan-Kolchin, M., Horiuchi, S., et al. 2016, MNRAS, 459, 1489

Breddels, M. A. \& Veljanoski, J. 2018, A\&A, 618, 13

Bullock, J. S. \& Boylan-Kolchin, M. 2017, ARA\&A, 55, 343

Carlberg, R. G. 2013, ApJ, 775

Carlberg, R. G. \& Grillmair, C. J. 2013, ApJ, 768, 171

Dalal, N. \& Kochanek, C. S. 2002, ApJ, 572, 25

Davis, M., Efstathiou, G., Frenk, C. S., \& White, S. D. M. 1985, ApJ, 292, 371

De Boer, T. J. L., Belokurov, V., Koposov, S. E., et al. 2018, MNRAS, 477, 1893

de Zeeuw, T. 1985, MNRAS, 216, 273

Dejonghe, H. \& de Zeeuw, T. 1988, ApJ, 333, 90

Diemand, J., Kuhlen, M., \& Madau, P. 2007, ApJ, 667, 859

Diemand, J., Kuhlen, M., Madau, P., et al. 2008, Nature, 454

D’Onghia, E., Springel, V., Hernquist, L., \& Keres, D. 2010, ApJ, 709, 1138

Eilers, A.-C., Hogg, D. W., Rix, H.-W., \& Ness, M. K. 2019, ApJ, 871, 120

Erkal, D. \& Belokurov, V. 2015a, MNRAS, 450, 1136

Erkal, D. \& Belokurov, V. 2015b, MNRAS, 454, 3542

Erkal, D., Belokurov, V., Bovy, J., \& Sanders, J. L. 2016, MNRAS, 463, 102

Erkal, D., Koposov, S. E., \& Belokurov, V. 2017, MNRAS, 470, 60

Famaey, B. \& Dejonghe, H. 2003, MNRAS, 340, 752

Gaia Collaboration, Brown, A. G. A., Vallenari, A., et al. 2018, A\&A, 616, 21

Gaia Collaboration, Prusti, T., J de Bruijne, J. H., et al. 2016, A\&A, 595, A1

Goldstein, H., Poole, C., Safko, J., \& Addison, S. R. 2002, Classical Mechanics,

3rd edn. (New York: Addison-Wesley), 638

Grillmair, C. J. \& Dionatos, O. 2006, ApJ, 643, L17

Harris, W. E. 1996, AJ, 112, 1487

Helmi, A. \& Gomez, F. 2007, ArXiv, 0710.0514, 10

Helmi, A. \& Koppelman, H. H. 2016, ApJ, 828, L10

Helmi, A. \& White, S. D. M. 1999, MNRAS, 307, 495

Helmi, A., White, S. D. M., De Zeeuw, P. T., et al. 1999, Nature, 402, 53

Hsueh, J. W., Enzi, W., Vegetti, S., et al. 2020, MNRAS, 492, 3047

Hu, W., Barkana, R., \& Gruzinov, A. 2000, Phys. Rev. Lett., 85, 1158

Hui, L., Ostriker, J. P., Tremaine, S., \& Witten, E. 2017, Phys. Rev. D, 95

Hunter, J. D. 2007, Computing in Science \& Engineering, 9, 90

Ibata, R., Thomas, G., Famaey, B., et al. 2020, ApJ, 891, 161

Ibata, R. A., Lewis, G. F., Irwin, M. J., \& Quinn, T. 2002, MNRAS, 332, 915

Ibata, R. A., Malhan, K., \& Martin, N. F. 2019, ApJ, 872, 152

Johnston, K. V., Spergel, D. N., \& Haydn, C. 2002, ApJ, 570, 656

Kluyver, T., Ragan-Kelley, B., Pérez, F., et al. 2016, Jupyter Notebooks-a pub-

lishing format for reproducible computational workflows (IOS Press)

Klypin, A., Kravtsov, A. V., Valenzuela, O., \& Prada, F. 1999, ApJ, 522, 82

Li, T. S., Koposov, S. E., Erkal, D., et al. 2020, arXiv, 2006.10763, 31

Malhan, K., Ibata, R. A., \& Martin, N. F. 2018, MNRAS, 481, 3442

Moore, B., Ghigna, S., Governato, F., et al. 1999, ApJ, 524, L19

Neto, A. F., Gao, L., Bett, P., et al. 2007, MNRAS, 381, 1450

Odenkirchen, M., Grebel, E. K., Rockosi, C. M., et al. 2001, ApJ, 548, L165

Pearson, S., Price-Whelan, A. M., \& Johnston, K. V. 2017, Nature Astronomy, 1,633

Peterson, P. 2009, Int. Journal of Computational Science and Engineering, 4, 296

Price-Whelan, A. M. \& Bonaca, A. 2018, ApJ, 863, L20

Reino, S., Rossi, E. M., Sanderson, R. E., et al. 2020, arXiv, 2007.00356

Ritondale, E., Vegetti, S., Despali, G., et al. 2019, MNRAS, 485, 2179

Sanders, J. L. \& Binney, J. 2015, MNRAS, 447, 2479

Sanders, J. L., Bovy, J., \& Erkal, D. 2016, MNRAS, 457, 3817

Sanderson, R. E., Vera-Ciro, C., Helmi, A., \& Heit, J. 2016, arXiv, 1608.05624

Sawala, T., Pihajoki, P., Johansson, P. H., et al. 2017, MNRAS, 467, 4383

Shipp, N., Drlica-Wagner, A., Balbinot, E., et al. 2018, ApJ, 862, 114

Shipp, N., Li, T. S., Pace, A. B., et al. 2019, ApJ, 885, 3

Spergel, D. N. \& Steinhardt, P. J. 2000, Phys. Rev. Lett., 84,

Springel, V. 2005, MNRAS, 364, 1105

Springel, V., Wang, J., Vogelsberger, M., et al. 2008, MNRAS, 391, 1685

Thomas, G., Ibata, R., Famaey, B., Martin, N., \& Lewis, G. 2016, MNRAS, 460 2711

Thompson, R. 2014, Astrophysics Source Code Library, record ascl:1411.001

Van Der Walt, S., Colbert, S. C., \& Varoquaux, G. 2011, Computing in Science and Engineering, 13, 22

Vegetti, S., Koopmans, L. V. E., Bolton, A., Treu, T., \& Gavazzi, R. 2010, MNRAS, 408

Vegetti, S., Lagattuta, D. J., McKean, J. P., et al. 2012, Nature, 481, 341

Vogelsberger, M., White, S. D. M., Helmi, A., \& Springel, V. 2008, MNRAS, 385,236

Vogelsberger, M., Zavala, J., Cyr-Racine, F.-Y., et al. 2016, MNRAS, 460, 1399

Yoon, J. H., Johnston, K. V., \& Hogg, D. W. 2011, ApJ, 731, 58

Zhu, Q., Marinacci, F., Maji, M., et al. 2016, MNRAS, 458, 1559 


\section{Appendix A: Covariance matrix - full 3D impulse}

In this section we derive the change of the covariance matrix when the full 3D morphology of the stream is taken into account. Similar to the 1D case, see Sec. 2.5. we assume that the change in velocity is a linear function of the spatial coordinates, meaning the denominator of the kicks $\left(w\left(\left(r_{s}^{2}+r^{2}\right) w^{2}-(\boldsymbol{x} \cdot \boldsymbol{w})^{2}\right)\right) \approx r_{s}^{2} w^{3}$. This approximation is in general true for the small volumes in which we measure the density, typically $<<1 \mathrm{kpc}$. This assumption allows us to rewrite Eqs. (7) to

$\Delta v_{i}(\boldsymbol{x})=-2 G M \frac{w^{2} x_{i}-w_{i}(\boldsymbol{x} \cdot \boldsymbol{w})}{r_{s}^{2} w^{3}}$,

where the subscript $i=x, y, z$.

In a similar procedure as for the 1D approximation we can now compute the covariance terms. The velocity-position terms in the most general form are

$C\left(v_{i}+\Delta v_{i}, x_{j}\right)=C\left(v_{i}, x_{j}\right)+\frac{1}{n} \sum \Delta v_{i}\left(x_{j}-\bar{x}_{j}\right)$

with

$\frac{1}{n} \sum \Delta v_{i}\left(x_{j}-\bar{x}_{j}\right)=-\frac{2 G M}{r_{s}^{2} w^{3}}\left[w^{2} C\left(x_{i}, x_{j}\right)-\sum_{k=x, y, z} w_{i} w_{k} C\left(x_{k}, x_{j}\right)\right]$.

The velocity-velocity terms are a bit more cumbersome

$C\left(v_{i}+\Delta v_{i}, v_{j}+\Delta v_{j}\right)=C\left(v_{i}, v_{j}\right)+\frac{1}{n} \sum \Delta v_{i}\left(v_{j}-\bar{v}_{j}\right)+\frac{1}{n} \sum\left(v_{i}-\bar{v}_{i}\right) \Delta v_{j}+\frac{1}{n} \sum \Delta v_{i} \Delta v_{j}$

where

$\frac{1}{n} \sum \Delta v_{i}\left(v_{j}-\bar{v}_{j}\right)=-\frac{2 G M}{r_{s}^{2} w^{3}}\left[w^{2} C\left(x_{i}, v_{j}\right)-\sum_{k=x, y, z} w_{i} w_{k} C\left(x_{k}, v_{j}\right)\right]$.

The last term in Eq. A.4 is

$$
\begin{aligned}
\frac{1}{n} \sum \Delta v_{i} \Delta v_{j}=\left(\frac{2 G M}{r_{s}^{2} w^{3}}\right)^{2}\left[w^{4} C\left(x_{i}, x_{j}\right)\right. & -\sum_{k=x, y, z}\left(w^{2} w_{k}\left[w_{j} C\left(x_{i}, x_{k}\right)+w_{i} C\left(x_{j}, x_{k}\right)\right]-w_{i} w_{j} w_{k}^{2} C\left(x_{k}, x_{k}\right)\right) \\
& \left.+w_{i} w_{j}\left(2 w_{x} w_{y} C(x, y)+2 w_{x} w_{z} C(x, z)+2 w_{y} w_{z} C(y, z)\right)\right] .
\end{aligned}
$$

These expressions take a much simpler form if the initial covariance matrix $\Sigma_{\varpi, 0}$ (e.g. Eq. 19] is diagonal. In this case, Eq. (A.4) simplifies to

$\frac{1}{n} \sum \Delta v_{i}\left(x_{j}-\bar{x}_{j}\right)=-\epsilon \frac{w}{r_{s}} C\left(x_{j}, x_{j}\right)\left[\delta_{i j}-\frac{w_{i} w_{j}}{w^{2}}\right]$

where

$\epsilon=\frac{2 G M}{r_{s} w^{2}}$

is a unit less parameter and $\delta_{i j}$ is the Kronecker delta. Equation A.5 simply vanishes, since it only features off-diagonal terms, and Eq. A.6 reduces to

$\frac{1}{n} \sum \Delta v_{i} \Delta v_{j}=\epsilon^{2} w^{2}\left[\frac{C\left(x_{i}, x_{j}\right)}{r_{s}^{2}}-\frac{w_{i} w_{j}}{w^{2} r_{s}^{2}}\left[C\left(x_{i}, x_{i}\right)+C\left(x_{j}, x_{j}\right)\right]+\sum_{k=x, y, z}\left(\frac{w_{i} w_{j} w_{k}^{2}}{w^{4}} \frac{C\left(x_{k}, x_{k}\right)}{r_{s}^{2}}\right)\right]$.

In Appendix C we will turn back to these simplified expressions. It will be convenient to express Eqs. (A.7) and A.9 in terms of $\epsilon$ and another parameter (respectively $\Delta_{i j}$ and $D_{i j}$ ) that carries all other terms, such that in this specific case we can write

$\frac{1}{n} \sum \Delta v_{i}\left(x_{j}-\bar{x}_{j}\right)=-\epsilon \Delta_{i j}$

and

$\frac{1}{n} \sum \Delta v_{i} \Delta v_{j}=\epsilon^{2} D_{i j}$ 


\section{Appendix B: Subhalo scaling relations}

For the scaling of the subhalos scale radius $r_{s}$ with mass $M$ we have used several scaling relations, they are listed below. To obtain the scaling we first relate the subhalo mass $M$ with the maximum circular velocity $V_{\max }=V_{c}\left(r_{\max }\right)$. In Springel et al. (2008) (see their Fig. 27) we find

$V_{\max }=\left(\frac{M}{3.37 \cdot 10^{7} \mathrm{M}_{\odot}}\right)^{1 / 3.49} \cdot 10 \mathrm{~km} / \mathrm{s}$,

which is an empirical scaling relation based on the subhalos down to the mass-range of $\lesssim 10^{5} \mathrm{M}_{\odot}$, identified in the 'Aquarius' simulations. Next, $r_{\max }$ is related to $V_{\max }$ using Eqs. $(6,8,9)$ from Springel et al.

$r_{\max }=V_{\max }\left[\frac{\delta_{c} H_{0}^{2}}{14.426}\right]^{-\frac{1}{2}} \cdot 0.62$

where the factor 0.62 is added based on the comment in the caption of Fig. 26 of Springel et al. (2008, see also below) and we assume $H_{0}=73 \mathrm{~km} / \mathrm{s} / \mathrm{Mpc}$. The final missing piece is $\delta_{c}$, which is related to the concentration parameter $c$ by

$\delta_{c}=\frac{200}{3} c^{3}\left(\log (1+c)-\frac{c}{1+c}\right)^{-1}$.

Typically $c$ is related to the subhalo mass $M$, motivated by Springel et al. (2008), we relate the two using an empirical scaling relation found by Neto et al. (2007) for relaxed halos

$c=5.26\left(\frac{M}{h \cdot 10^{14}}\right)^{-0.10}$,

where $h=H_{0} /(100 \mathrm{~km} / \mathrm{s})$. We note that Springel et al. (2008) find that the resulting scaling relation of $V_{\max } \sim r_{\max }$ is lower than the relation found from extrapolating the results of Neto et al. (which is not calibrated for subhalos in the low-mass range that we consider here). The offset is 0.62 , which is why we add this factor in Eq. (B.2).

With the equations above we can relate the subhalo mass $M$ to $V_{\max }$ and a corresponding $r_{\max }$. The scale radius $r_{s, \mathrm{NFW}}$ is related to $r_{\max }$ simply as

$r_{\max }=2.163 \cdot r_{s, \mathrm{NFW}}$

which is found numerically from calculating where $V_{c}\left(r_{\max }\right)=V_{\max }$ (but see Eq. (11) of Diemand et al. (2007), where we originally found the relation).

Finally, in this main text we use a Plummer profile to describe the subhalos, rather than an NFW. Therefore, we relate the scale radii of the two profiles by equation the acceleration at $r_{\max }$

$a_{\mathrm{NFW}}\left(r_{\max }\right)=a_{\text {Plummer }}\left(r_{\max }\right)=-\frac{G M}{\left(r_{\max }^{2}+r_{s}^{2}\right)^{3 / 2}} \cdot r_{\max }$

The scale radius of the Plummer, $r_{s}$, can be found by solving the equation above, which then is a function of $M$ only. Finally, by fitting the scaling relation numerically we find that the scale radius depends on mass by $r_{s} \propto M^{0.397} \sim M^{2 / 5}$.

\section{Appendix C: Computation of the density contrast at late times}

As described in the main paper, the density contrast at late times takes the form (see Eq. 34 )

$\delta \rho_{\mathrm{str}}^{\mathrm{gap}}=\sqrt{\frac{\operatorname{det}\left|\sigma_{\Theta_{0}}\right|_{\text {str }}}{\operatorname{det}\left|\sigma_{\Theta_{0}}\right|_{\text {gap }}}}$.

To be able to establish its dependence on the characteristic parameters of the encounter, we need to determine the form of determinant of the matrix $\sigma_{\Theta_{0}}$. This is the upper left, $3 \times 3$, submatrix of $\sigma_{\omega, 0}$ that is described in Sec. 2.5. This latter matrix, following the notation of Sec. 2.4, takes the form

$\sigma_{\omega, 0}=\mathcal{M}_{0}^{-1^{\dagger}} \sigma_{\varpi, 0} \mathcal{M}_{0}^{-1}$

where $\mathcal{M}_{0}$ is given by Eq. 12 , namely $\mathcal{M}_{0}=\mathcal{M}_{0}^{\mathrm{AA} \leftarrow \mathrm{st}} \mathcal{M}_{0}^{\mathrm{st} \leftarrow \mathrm{cyl}} \mathcal{M}_{0}^{\text {cyl } \leftarrow \text { xyz }}$, and thus represents the coordinate transformations from Cartesian to action-angle variables. For example the matrix that accounts for the transformation from Stäckel coordinates to action angles, $\mathcal{M}_{0}^{\mathrm{AA} \leftarrow \mathrm{st}}$, contains the derivatives of the characteristic function and its general form is given in Eq. (A2) of HW99. In Eq. (C.2), $\sigma_{\varpi}^{-1}=\Sigma_{\varpi}$ is the inverse of the $6 \times 6$ covariance matrix in Cartesian coordinates. Therefore, $\sigma_{\Theta_{0}}$ for the stream depends on location as well as on the initial properties of the stream, and similarly for the gap.

The matrix $\sigma_{\varpi, 0}$ is of the form

$\sigma_{\varpi, 0}=\left(\begin{array}{cc}\sigma_{x, 0} & \sigma_{x v, 0} \\ \sigma_{x v, 0}^{\dagger} & \sigma_{v, 0}\end{array}\right)$,

Article number, page 14 of 15 
and since we may express as

$\mathcal{M}_{0}^{-1}=\left(\begin{array}{ll}A & B \\ C & D\end{array}\right)$

this means that

$\sigma_{\Theta_{0}}=A^{\dagger} \sigma_{x, 0} A+C^{\dagger} \sigma_{x v, 0}^{\dagger} A+A^{\dagger} \sigma_{x v, 0} C+C^{\dagger} \sigma_{v, 0}^{\dagger} C$.

Using matrix inversions, if

$\mathcal{M}_{0}=\left(\begin{array}{ll}t_{1} & t_{2} \\ t_{3} & t_{4}\end{array}\right)$

and $t_{4}$ is invertible then

$A=\left(t_{1}-t_{2} t_{4}^{-1} t_{3}\right)^{-1}, \quad$ and $\quad C=-t_{4}^{-1} t_{3} A=T_{43} A$,

where recall that $A$ and $T_{43}$ represent coordinate transformations, and therefore depend only on location. These matrices have been set to be identical for the gap and the stream in Eq. [C.1], which significantly simplifies subsequent computations. If we now replace in Eq. C.5), this results in

$\operatorname{det}\left|\sigma_{\Theta_{0}}\right|=(\operatorname{det} A)^{2} \operatorname{det}\left(\sigma_{x, 0}+T_{43}^{\dagger} \sigma_{x v, 0}^{\dagger}+\sigma_{x v, 0} T_{43}+T_{43}^{\dagger} \sigma_{v, 0}^{\dagger} T_{43}\right)$

The expression for det $\left|\sigma_{\Theta_{0}}\right|_{\text {str }}$ using the above equation has been worked out in detail by HW99 for a stream generated from an initially isotropic Gaussian distribution in configuration and velocity space, and for a preferred location along the orbit, namely the apocentre. The reader may wish to check the explicit expressions in the case of an axisymmetric system (the last equation in their Appendix B), and for a system described with Stäckel coordinates (Eq. C13 in their Appendix C).

We now proceed to determine the form of the submatrices of $\sigma_{\varpi, 0}$ given in Eq. (C.3) and needed in Eq. (C.8). For the stream we will assume no initial correlations between positions and velocities in the stream (i.e. a diagonal covariance matrix $\Sigma_{\varpi, 0}$ ), which means that the submatrix $\sigma_{\boldsymbol{x}, 0}^{\text {str }}=0$, and that $\sigma_{\boldsymbol{x}, 0}^{\text {str }}=\left[\mathbb{C}_{\boldsymbol{x}, \boldsymbol{x}_{0}}^{\text {str }}\right]^{-1}$ and $\sigma_{\boldsymbol{v}, 0}^{\text {str }}=\left[\mathbb{C}_{\boldsymbol{v}, \boldsymbol{v}_{0}}^{\text {str }}\right]^{-1}$ according to Eq. [19]. For the gap, we may express

$\mathbb{C}_{\boldsymbol{v}, v_{0}}^{\mathrm{gap}}=\mathbb{C}_{\boldsymbol{v}, v_{0}}^{\mathrm{str}}+\epsilon^{2} \mathbf{D}$

where the elements of $\mathbf{D}$ are given by $D_{i j}$, see Eq. A.11. Furthermore,

$\mathbb{C}_{x, v_{0}}^{\text {gap }}=-\epsilon \Delta$

where the elements of $\Delta$ are given by $\Delta_{i j}$, see Eq. (A.10).

To compute the submatrices $\sigma_{\boldsymbol{x}, 0}^{\text {gap }}, \sigma_{\boldsymbol{x}, 0}^{\text {gap }}$ and $\sigma_{v, 0}^{\text {gap }}$, in Eq. C.8. we use that $\sigma_{\varpi, 0}^{\text {gap }}=\Sigma_{\varpi, 0}^{\text {gap }-1}$, which is given by Eq. 20, The inverse of this block matrix can be computed explicitly ${ }^{3}$ provided the matrix $W=\mathbb{C}_{\boldsymbol{v}, \boldsymbol{v}_{0}}^{\text {gap }}-\mathbb{C}_{\boldsymbol{x}, \boldsymbol{v}_{0}}^{\text {ga }}\left[\mathbb{C}_{\boldsymbol{x}, \boldsymbol{x}_{0}}^{\text {gap }}\right]^{-1} \mathbb{C}_{\boldsymbol{x}, \boldsymbol{v}_{0}}^{\text {gap }}$ is invertible. Using Eqs. (C.9) and (C.10), and to the lowest order in $\epsilon$ we find that

$$
\begin{aligned}
\sigma_{\boldsymbol{v}, 0}^{\text {gap }} & =W^{-1} \approx\left[\mathbb{C}_{\boldsymbol{v}, \boldsymbol{v}_{0}}^{\mathrm{str}}\right]^{-1}\left[\mathbf{I}-\epsilon^{2}\left(\mathbf{D}-\boldsymbol{\Delta}^{\dagger}\left[\mathbb{C}_{\boldsymbol{x}, \boldsymbol{x}_{0}}^{\mathrm{str}}\right]^{-1} \boldsymbol{\Delta}\right)\left[\mathbb{C}_{\boldsymbol{v}, \boldsymbol{v}_{0}}^{\mathrm{str}}\right]^{-1}\right], \\
\sigma_{\boldsymbol{x} \boldsymbol{v}, 0}^{\text {gap }} & =\epsilon\left[\mathbb{C}_{\boldsymbol{x}, \boldsymbol{x}_{0}}^{\mathrm{str}}\right]^{-1} \boldsymbol{\Delta}\left[\mathbb{C}_{\boldsymbol{v}, \boldsymbol{v}_{0}}^{\mathrm{str}}\right]^{-1} \\
\sigma_{\boldsymbol{x}, 0}^{\text {gap }} & =\left[\mathbb{C}_{\boldsymbol{x}, \boldsymbol{x}_{0}}^{\mathrm{str}}\right]^{-1}+\epsilon^{2}\left[\mathbb{C}_{\boldsymbol{x}, \boldsymbol{x}_{0}}^{\mathrm{str}}\right]^{-1} \boldsymbol{\Delta}\left[\mathbb{C}_{\boldsymbol{v}, \boldsymbol{v}_{0}}^{\mathrm{str}}\right]^{-1} \boldsymbol{\Delta}^{\dagger}\left[\mathbb{C}_{\boldsymbol{x}, \boldsymbol{x}_{0}}^{\mathrm{str}}\right]^{-1} .
\end{aligned}
$$

We are now almost ready to compute the density contrast, since

$\operatorname{det}\left|\sigma_{\Theta_{0}}\right|_{\text {gap }} \sim(\operatorname{det} A)^{2} \operatorname{det}\left[\sigma_{x, 0}^{\mathrm{str}}+T_{43}^{\dagger} \sigma_{v, 0}^{\mathrm{str}} T_{43}+\epsilon\left(T_{43}^{\dagger} \sigma_{v, 0}^{\mathrm{str}} \Delta^{\dagger} \sigma_{\boldsymbol{x}, 0}^{\mathrm{str}}+\sigma_{\boldsymbol{x}, 0}^{\mathrm{str}} \Delta \sigma_{v, 0}^{\mathrm{str}} T_{43}\right)\right] \sim \operatorname{det}\left|\sigma_{\Theta_{0}}\right|_{\text {str }} \operatorname{det}[\mathbf{I}+\epsilon \mathbf{K}]$,

where the matrix $\mathbf{K}$ is

$\mathbf{K}=\left[T_{43}^{\dagger} \sigma_{v, 0}^{\mathrm{str}} \Delta^{\dagger} \sigma_{x, 0}^{\mathrm{str}}+\sigma_{x, 0}^{\mathrm{str}} \Delta \sigma_{v, 0}^{\mathrm{str}} T_{43}\right]\left[\sigma_{x, 0}^{\mathrm{str}}+T_{43}^{\dagger} \sigma_{v, 0}^{\mathrm{str}} T_{43}\right]^{-1}$,

and it is therefore dependent on the initial properties of the stream (at the time of the encounter and through the $\sigma$ matrices), the location of the encounter in phase-space (through the matrix $T_{43}$ introduced in Eq. C.7), and the characteristic parameters of the encounter (through the matrix $\Delta$ whose elements can be described as $\frac{w}{r_{s}}$ times some function $g\left(\mathbb{C}_{\boldsymbol{x}, \boldsymbol{x}_{0}}^{\mathrm{str}}, \alpha, \theta\right)$, see Eqs. A.7 and A.10). Since we may express $\operatorname{det}[\mathbf{I}+\epsilon \mathbf{K}] \sim 1+\epsilon$ trK, the density contrast at late times becomes

$\delta \rho_{\mathrm{str}}^{\mathrm{gap}} \sim \sqrt{\frac{\operatorname{det}\left|\sigma_{\Theta_{0}}\right|_{\text {str }}}{\operatorname{det}\left|\sigma_{\Theta_{0}}\right|_{\mathrm{gap}}}} \sim(1+\epsilon \operatorname{tr} \mathbf{K})^{-1 / 2} \sim 1-\frac{\epsilon}{2} \operatorname{tr} \mathbf{K} \sim 1-\frac{G M}{r_{s}^{2} w} f\left(\theta, \alpha, \mathbb{C}_{\boldsymbol{x}, \boldsymbol{x}_{0}}^{\mathrm{str}}, \mathbb{C}_{\boldsymbol{v}, \boldsymbol{v}_{0}}^{\mathrm{str}}, \boldsymbol{x}_{0}, \boldsymbol{v}_{0}\right)$,

where $f\left(\theta, \alpha, \mathbb{C}_{\boldsymbol{x}, \boldsymbol{x}_{0}}^{\mathrm{str}}, \mathbb{C}_{\boldsymbol{v}, \boldsymbol{v}_{0}}^{\mathrm{str}}, \boldsymbol{x}_{0}, \boldsymbol{v}_{0}\right)$ is a function that depends on the impact configuration and initial state of the stream.

3 see https://en.wikipedia.org/wiki/Block_matrix\#Block_matrix_inversion and references therein 